\title{
Wing-Nacelle Assembly Multidisciplinary Performance Optimization
}

\author{
Yevgeniy M. Gisin ${ }^{*}$ and David D. Marshall ${ }^{\dagger}$ \\ California Polytechnic State University at San Luis Obispo, San Luis Obispo, CA, 93401
}

The paper analyzes the aerodynamics benefits of above-wing mounting of turbofan engines and performs a trade-off study of the structural limitations with this mounting method. This research is based on the US Patent \#6,308,913, aiming to quantify the actual benefit derived from the drag reduction measure described in the patent. The paper focuses on the analysis of a turbofan engine, which when installed above an aircraft's wing creates a flow field optimized to reduce the overall drag experienced by the wing nacelle assembly. This installation is designed to reduce wave drag during transonic cruise and therefore the wing-nacelle geometry was analyzed at a $C_{L}$ value of 0.4 and Mach number of 0.75 . An aerodynamically ideal location for the nacelle was ascertained, and drag characteristics calculated. A multidisciplinary optimization study, considering the effects of drag, structural weight and aircraft mission was performed. This study does not, and was not intended to have the fidelity necessary for detailed design of an airframe-engine installation, but is instead intended to provide guidelines for use in the preliminary-design field.

\section{Nomenclature}

\begin{tabular}{|c|c|c|}
\hline \multicolumn{3}{|c|}{ Abbreviations } \\
\hline$A O A$ & $=$ & Angle of attack \\
\hline$c \# r \#$ & $=$ & Nacelle Location in \%MAC and \# of nacelle radii \\
\hline$C F D$ & $=$ & Computational Fluid Dynamics \\
\hline$C_{L}$ & $=$ & Coefficient of Lift \\
\hline FOD & $=$ & Foreign Object Damage \\
\hline$G$ & $=$ & Gravity \\
\hline$L / D$ & $=$ & Lift-to-Drag Ratio \\
\hline$\angle B O$ & $=$ & Local Box of Interest \\
\hline$L E$ & $=$ & Leading Edge \\
\hline$M A C$ & $=$ & Mean Aerodynamic Chord \\
\hline$M D O$ & $=$ & Multi-Disciplinary Optimization \\
\hline$G C I$ & $=$ & Grid Convergence Index \\
\hline GMRES & $=$ & Generalized Minimal RESidual algorithm \\
\hline \multicolumn{3}{|l|}{ Symbols } \\
\hline$C_{d}$ & $=$ & Drag coefficient \\
\hline$C_{f}$ & $=$ & Friction coefficient \\
\hline$f$ & $=$ & Value of solution \\
\hline$H$ & $=$ & Shape factor \\
\hline$H^{*}$ & $=$ & Shape factor: Kinetic energy thickness/Momentum loss thickness \\
\hline$H^{* *}$ & $=$ & Shape factor: Density thickness/Momentum loss thickness \\
\hline$M$ & $=$ & Mach number, Unit vector in network row direction \\
\hline$N$ & $=$ & Unit vector in network column direction \\
\hline$\hat{n}$ & $=$ & Normal vector \\
\hline$p$ & $=$ & Observed order of convergence \\
\hline
\end{tabular}

\footnotetext{
* Masters Student, Department of Aerospace Engineering, 1 Grand Ave., San Luis Obispo, CA 93401, AIAA Student Member.

${ }^{\dagger}$ Assistant Professor, Department of Aerospace Engineering, 1 Grand Ave., San Luis Obispo, CA 93401, AIAA Member. 


$\begin{array}{lll}Q & =\text { Source } \\ r & =\text { Grid refinement ratio } \\ S & =\text { AGPS parametric direction 1 } \\ T & =\text { AGPS parametric direction } 2 \\ u, v, w & =\text { Mass flux vector } \\ \vec{W} & =\text { Free-stream flow direction } \\ X & =\text { Green's function } \\ \xi & =\text { Density } \\ \rho & =\text { Momentum loss thickness } \\ \theta & =\text { Continuous far field (Prandtl-Glauert) operator } \\ \tau & =\text { Total potential } \\ \Phi & & \\ \nabla & = & \text { Solution grid density 1=highest } \\ \text { Subscripts } & =\text { Drag } \\ 1,2,3 & =\text { Solution at infinite grid density } \\ D & =\text { Lift } \\ e & =\text { Time } \\ h=0 & & \end{array}$

\title{
I. Introduction
}

\begin{abstract}
A wide variety of air intake geometries have found use on modern turbojet-powered aircraft, with each type having its benefits and disadvantages. In all conventional engine installations the designer attempts to reduce the normally harmful effects of the aerodynamic interaction between the nacelle and the wing. Because of this consideration, most modern engines are installed either in nacelles separate from the aircraft body or use intakes located either below or to the side of the aircraft body. When considering the distinct set of challenges facing the designer of a small passenger jet aircraft, an engine mounting location on the upper body of the aircraft gains a number of significant benefits. Since there is no need to create space for wing-mounted engines underneath the aircraft, a short main landing gear can be used. The reduction in aircraft height also makes it better suited for field operations such as servicing and passenger loading. Most popular variant of such an installation is seen on the majority of today's business jets - with the engines located on pylons at the rear of the fuselage. However, another approach is to install the engines on pylons raised above the wing surface. The main reason for this engine installation is the reduction of trans-sonic wave drag on the top wing surface. ${ }^{1}$ The flow field of the intake is superimposed on that of the wing, reducing the negative pressure peak and retarding the generation of a shock wave, thereby increasing the aircraft's divergence drag number. This engine installation brings a number of other advantages to the aircraft configuration - short and simple landing gear, lack of FOD ingestion issues, and a larger cabin volume afforded by the lack of an engine carrythrough.

This research explores an above-wing engine installation similar to that discussed in the Patent \#6,308,913 at a single Mach number. A set of aircraft wing geometries with an upper-surface mounted engine intake are evaluated in the Boeing TranAir potential flow CFD code. The research is conducted at a pre-assigned "cruise" condition for a notional aircraft, defined by Mach $=0.75$ and $\mathrm{C}_{\mathrm{L}}=0.4$. These values are used in order to conduct this research in conditions similar to those experienced by a hypothetical business jet aircraft during the cruise portion of its mission. Although an engine intake location highly-separated from the wing surface may derive the highest benefit from the supersonic aerodynamics viewpoint, this study attempts to optimize the engine location by also evaluating a structural constraint, which naturally acts to prohibit an excessively complicated engine mounting scheme. Using these two criteria, an optimum intake location is defined.
\end{abstract}

\section{Research Scope and Definition}

The study to find optimum wing performance focuses on the effects of varying the $\mathrm{X}$ and $\mathrm{Z}$ coordinate location of the nacelle in respect to the wing surface. The Y coordinate is kept constant throughout the study, and in order to simplify the amount of parameters affecting the trade study, no aircraft fuselage effects are considered or included. 
Having specified a notional aircraft configuration, the cruise angle of attack, and wing area are both determined. Because the cruise condition is where the aircraft's efficiency is most important, the engine location is optimized to reduce transonic drag at that point. In order to produce more generalized aerodynamic results, it was decided to not sweep either the leading or the trailing edge of the geometry analyzed in the TranAir.

The general process of the study undertaken can be explained by the flowchart presented in Figure 1.

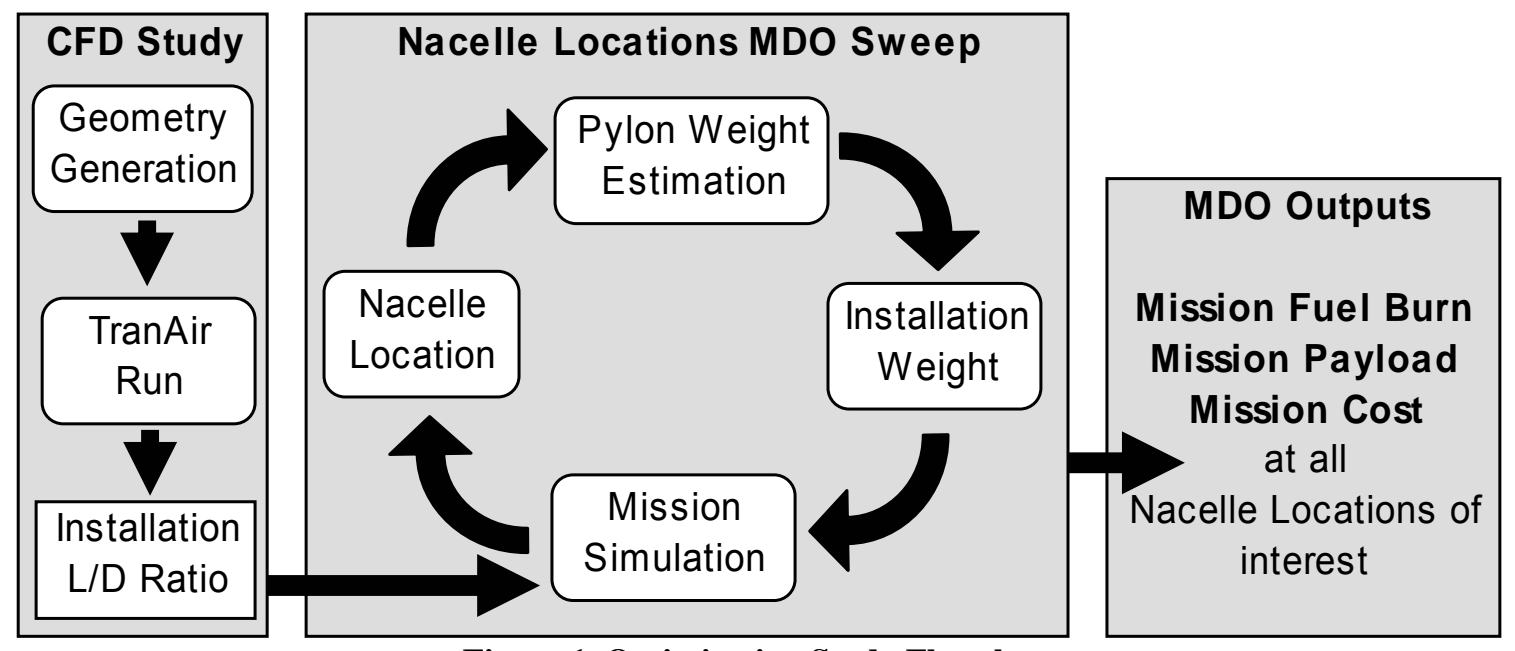

Figure 1. Optimization Study Flowchart

During the CFD study a set of wing-nacelle geometries defined by a range of nacelle locations is evaluated at a $\mathrm{C}_{\mathrm{L}}$ of 0.4 and a freestream Mach number of 0.75 to obtain $\mathrm{L} / \mathrm{D}$ values at each nacelle location point. The operations shown in the MDO section of the flowchart above are performed within a user-specified nacelle position range. The optimization routine sweeps all the possible nacelle locations of interest, which are normally limited by the range of available CFD data. Using a mission simulation routine at each incremental nacelle position, the benefits to the notional aircraft are obtained in real-world terms such as change in mission range, payload or operations cost. From an evaluation of these aircraft performance metrics, an optimum overall nacelle location point can be determined.

\section{CFD Tools Description}

The ready availability of the Boeing TranAir (A633) code on CalPoly campus, and the number of advanced features incorporated in this code made it an attractive choice. Automatic grid refinement, integral calculation of moderately turbulent boundary layers and the fast run-times offered by this code were all considered beneficial to conducting an accurate and efficient study. One of the main distinguishing features of TranAir is its use of a rectangular configuration grid superimposed over the configuration surface. This allows for a simple grid generation procedure, thus simplifying and speeding up the code execution.

\section{Solver Fundamentals}

The nonlinear, full-potential flow equation is the basis of the TranAir code, with the flow outside the boundary layer therefore being assumed to be inviscid, and irrotational. In potential flow, the flow velocity is assumed to be derivable from a scalar velocity potential $\Phi$, which is in turn defined as the gradient of the local velocity.

$$
\vec{U}=\vec{\nabla} \Phi[1]
$$

The full-potential equation, is then defined in terms of the unknown scalar velocity potential $\Phi$ in the following manner ${ }^{2}$.

$$
\frac{\partial(\rho u)}{\partial x}+\frac{\partial(\rho v)}{\partial y}+\frac{\partial(\rho w)}{\partial z}=0
$$

Because of the inability to achieve an analytical solution to the full-potential flow problem with a set of reasonable boundary conditions, TranAir, as all other CFD codes, discretizes the solution field by a set of nonlinear equations, and solves those. The solution field is split into a global grid of rectangular boxes (cells), each of which is then refined hierarchically to approach a solution with an error level specified by the user. TRANAIR solves a set of nonlinear equations which approximate the full-potential equation using the Newton method at each corner point of 
every cell of the grid. In applying the Newton method, each problem is linearized and the linear problem is solved during each iterative step. The linear problem is also solved iteratively using the GMRES method. ${ }^{3}$

In the TranAir implementation, the boundary layer solver is coupled to the inviscid solver, and the solution information is shared between the two codes. Two boundary layer codes, A411 and ISES, are incorporated in TranAir, the first a "loosely coupled", and the second a "closely coupled" boundary layer code. A411 passes information back and forth between the viscous and inviscid programs at each Newton step in the solution process, while the ISES code actually combines the equations for viscous and inviscid flows, and solves them all together. This coupling method provides the ISES code with improved solution convergence and allows it to achieve solutions for mildly separated flows, in addition to attached flows. Because ISES code was chosen to be used for boundary layer analysis in this study, the description will focus on this code.

The ISES 2-d boundary layer code was developed by Mark Drela and Michael B. Giles ${ }^{4}$; the newest version of it is marketed under the name MSES. A streamline-based Euler discretization and a two-equation boundary layer formulation are coupled through the displacement thickness and solved simultaneously by a full Newton method. ${ }^{5}$ ISES implements advanced transition and separation calculations with the capability to model laminar separation bubbles and limited zones of turbulent separation.

At its core, ISES uses a simplified form of the Prandtl boundary layer equations of continuity and conservation of momentum and energy.

$$
\begin{gathered}
\text { Continuity: } \frac{\partial \rho}{\partial t}+\frac{\partial}{\partial x}(\rho u)+\frac{\partial}{\partial y}(\rho v)=0 \\
\text { Momentum: } \rho\left(\frac{\partial u}{\partial t}+u \frac{\partial u}{\partial x}+v \frac{\partial u}{\partial y}\right)=-\frac{\partial p_{e}}{\partial x}+\frac{\partial}{d y}\left(\mu \frac{\partial u}{\partial y}\right) \\
\text { Energy: } \rho\left(\frac{\partial h}{\partial t}+u \frac{\partial h}{\partial x}+v \frac{\partial h}{\partial y}\right)=\frac{\partial p_{e}}{\partial t}+u \frac{\partial p_{e}}{\partial x}+\frac{\partial}{\partial y}\left(k \frac{\partial T}{\partial y}\right)+\mu\left(\frac{\partial u}{\partial y}\right)^{2}
\end{gathered}
$$

Assuming a perfect gas in steady flow and eliminating the velocity terms in the equations, integration can be performed outward from the wall to infinity. Finally, neglecting cross-stream pressure variation and considering only adiabatic freestreams, the final form of equations used in the program are achieved. ${ }^{6}$

$$
\begin{gathered}
\frac{d \theta}{d \xi}+\left(H+2-M_{e}^{2}\right) \frac{\theta}{u_{e}} \frac{d u_{e}}{d \xi}=\frac{C f}{2} \\
\theta \frac{d H^{*}}{d \xi}+\left(2 H^{* *}+H^{*}(1-H)\right) \frac{\theta}{u_{e}} \frac{d u_{e}}{d \xi}=2 C_{D}-H^{*} \frac{C f}{2}
\end{gathered}
$$

The boundary layer is described with a two-equation [6,7] lagged dissipation integral BL formulation and

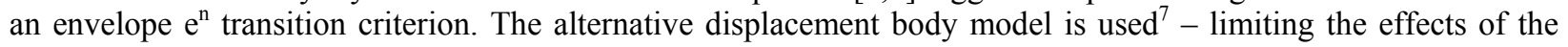
boundary layer to displacing the inviscid flow away from the physical body constraints. The boundary layer solution is calculated for a 2-d "rib" that is user-defined on top of the configuration geometry. In the TranAir application, the 3 -d solution is then interpolated from the 2-d ribs onto either an infinite swept wing with taper, or a body of revolution.

\section{Geometry Generation}

The baseline geometry created in AGPS is comprised of an axisymmetric nacelle separated from an unswept wing surface. This geometry is comprised of 5 surface networks and 3 viscous wake networks, which can all be seen in Figure 2. 


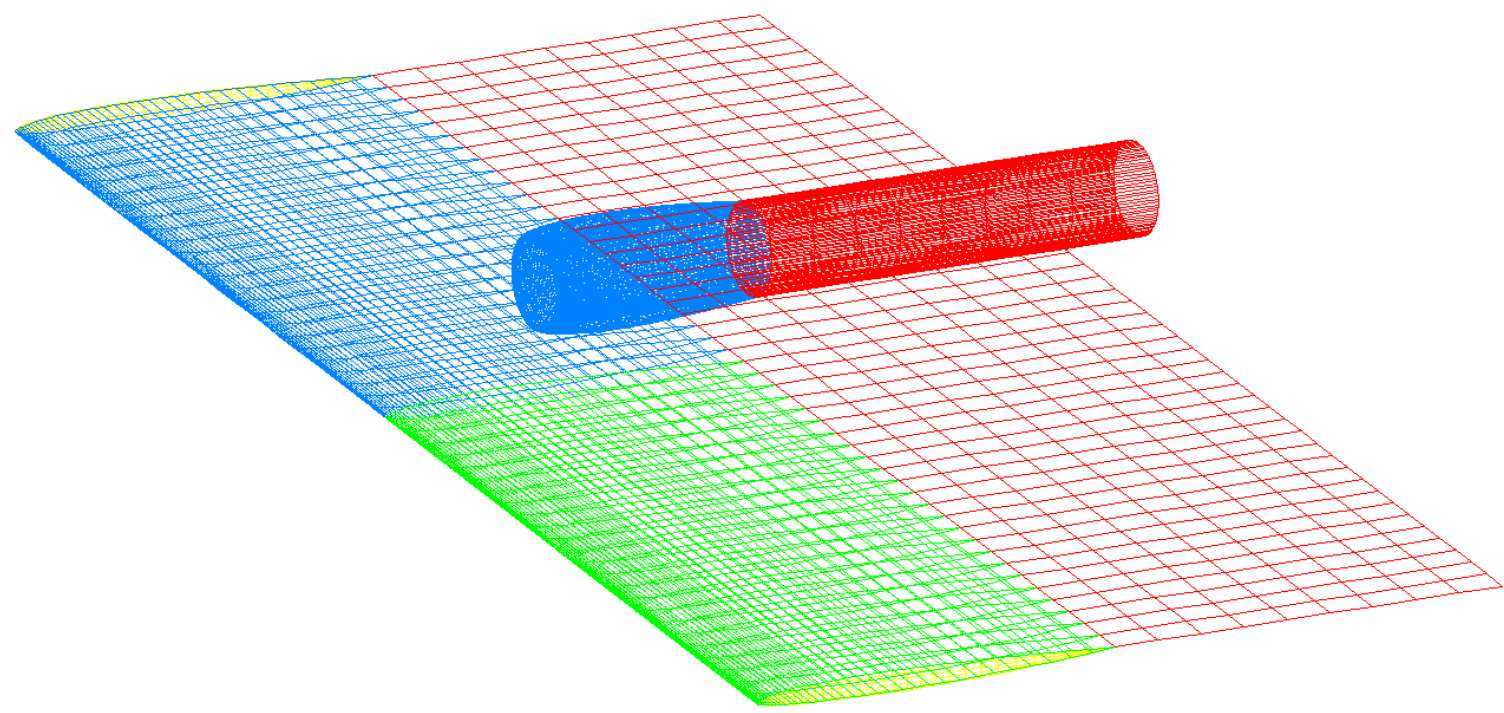

Figure 2 - Sample Geometry

A variety of different types of networks are available for use in order to allow for the representation of the different required types of boundary conditions. In the Figure 2 above, the viscous wake networks are shown in red. The single type-1 wraparound network making up the nacelle is outlined in blue. The two rounded wing cap networks are outlined in yellow. The two networks making up the left and right wing sections are shown in green and blue, respectively.

Table 1 - Networks Used

\begin{tabular}{|l|c|c|c|}
\hline & Grid Size (M:N) & Network Names & $\begin{array}{c}\text { Gridding type } \\
\text { (Row:Column) }\end{array}$ \\
\hline Nacelle & $101: 100$ & ng & uniform:custom \\
\hline Wing Surfaces & $81: 25$ & wrg, wlg & custom:uniform \\
\hline Wing Caps & $41: 7$ & rwcr, rwcl & custom:uniform \\
\hline Viscous Wakes & $10: 100,10: 25,10: 25$ & ngvw, wrvw, wlvw & uniform:uniform \\
\hline
\end{tabular}

The relevant dimensional parameters of the geometry used in the TranAir study are presented in Figure 3 below. To conduct the study at the full-scale wing Reynolds number of 10,000,000, a Reynolds number of 4,000,000 per unit length of TranAir geometry is specified in the boundary layer input section of the input file. The scaling between the CFD and real-life geometry is accomplished using the ratio of $2.085 \mathrm{ft} / \mathrm{unit}$. 


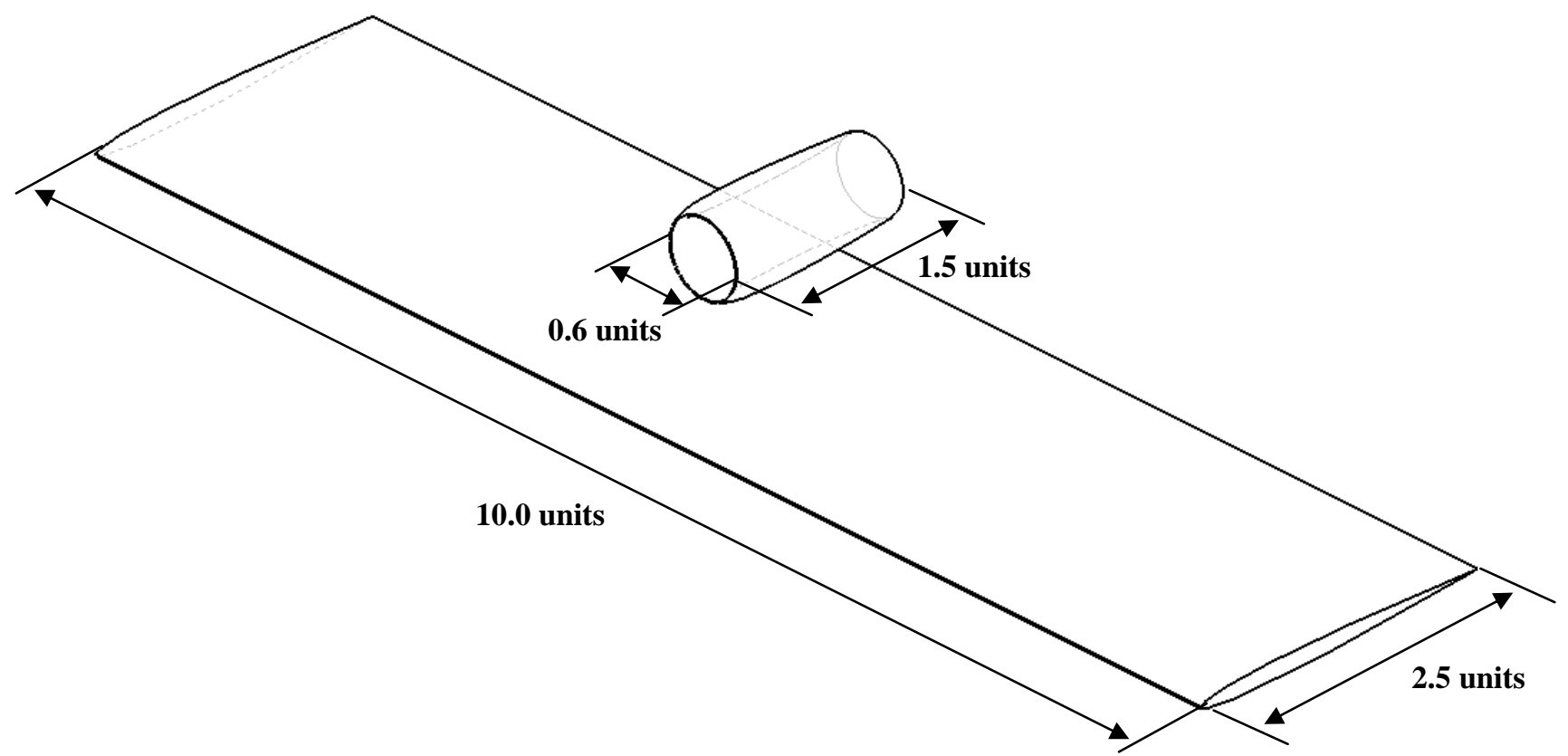

Figure 3. Geometry Analyzed in TranAir

\section{Structural Constraint}

This method was developed in an attempt to use the weight data available for aircraft with similar pylon engine installations. The underlying hypothesis in creating this method was that the weight of a pylon installation was mainly driven by the weight of the engine it supports, and by the length of the pylon. By correlating the structural weight of the "nacelle group" from a MIL-1374 weight breakdown form ${ }^{8}$ to the two pylon weight drivers mentioned above, Figure 4 was obtained. 


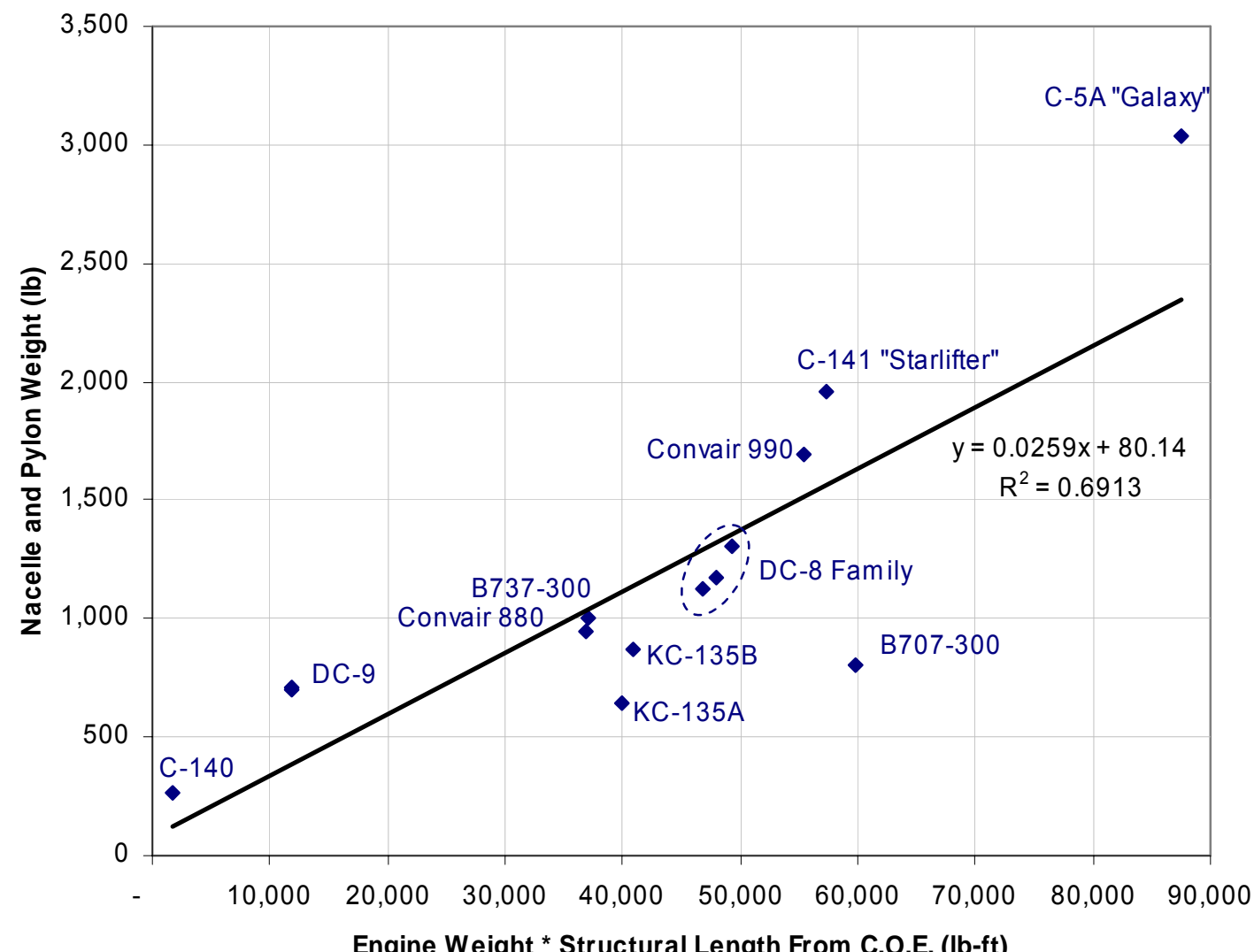

Figure 4. Pylon Weight Trendline

This trendline gives an added benefit of accounting for nacelle structure and other components necessary. The aircraft included in the study all have similar cruise speeds and mission requirements as the hypothetical aircraft. Aircraft with special mission characteristics that may impact the weight of the pylon installation, such as, for example, the A-10 ground attack aircraft, have not been used. The linear trendline drawn through the data points was used at each step of the MDO process to establish the weight of the nacelle + pylon combination on the hypothetical aircraft configuration.

\section{Multi Disciplinary Optimization Tool}

The Multi Disciplinary Optimization (MDO) tool allows for an optimizational synthesis of the results obtained during the CFD and structural studies, and showed the impact of various nacelle mounting options on an aircraft's performance during a given mission. A relatively simple flight simulation that limits its scope to the long-distance cruise component of an aircraft's mission is conducted, allowing the user to evaluate the amount of fuel burned by the aircraft. The tool was created in Microsoft Excel, and the important assumptions used in the construction of this tool are listed below.

The particular mission the hypothetical aircraft is being optimized for is $2,500 \mathrm{nmi}$ long, with the in-cruise specific fuel consumption of $0.5 \mathrm{lb} / \mathrm{lb} /$ hour being assumed. The empty weight of the aircraft is calculated as a combination of structural empty weight of 7,500lbs, added to the weight of the engine and installation. An engine similar in size to the Williams FJ-44 is assumed, with a dry weight of $445 \mathrm{lbf}$ used.

The CFD results map obtained earlier is input in a tabulated format into the MDO spreadsheet, where 3-d parabolic interpolation enabled by the XIX $\operatorname{trFun}^{9}$ Microsoft Excel plug-in allows the L/D value to be obtained at any hypothetical nacelle location. With the aircraft empty weight and L/D ratio determined, thrust required and fuel burn during cruise can be calculated. By subtracting total fuel used during the mission and aircraft empty weight from the takeoff gross weight, the maximum possible payload weight is derived. This payload weight is used as a metric of the aircraft's performance, since it can be used to either extend the flight range or to carry extra income-generating payload. 
The L/D ratio values are compared without taking into account the possible changes to the aircraft's fuselage and empennage. It is be assumed that the influence of these components will not vary between different nacelle geometries evaluated, and as such will decrease the overall L/D ratio of the all geometries by the same amount, therefore not affecting the result of the trade-off performed. The additional drag somewhat "dampens" the wing's aerodynamic benefit to the aircraft, and is compensated for by the assumption that wing drag constitutes only $50 \%$ of overall aircraft drag.

It should be mentioned that the MDO tool does not attempt to resize the aircraft in order to take the maximum advantage of a reduced fuel burn. Instead, with all aircraft configurations flown at the same overall gross takeoff weight, an assumption is made that a lower-fuel burn aircraft will carry extra payload in the space afforded to it by its lower structure weight. A full aircraft sizing process would require for an established relationship between engine thrust and weight in order to allow for weight estimation of engines of varied required thrust level. This relationship would introduce another level of uncertainty to the results of the study, and was judged to be excessive. In the current MDO implementation the gross takeoff weight of the aircraft stays constant, and therefore the mission chosen will not affect the optimum nacelle location.

By iterating through the applicable study range, it is possible to obtain the empty weight, takeoff gross, and inmission fuel consumption for all aircraft configurations of interest. This, in turn allows finding an aircraft configuration in which the benefit of an aerodynamically efficient engine installation and the drawback of the higher-weight structure required to achieve such an installation are balanced, providing the aircraft with the optimum in-mission performance.

\section{Results and Discussion}

\section{A. Grid Independence Study}

A grid independence study is performed in order to establish the correct degree of precision to use for the surface mesh networks. With the increase of surface mesh density the value of the flow solution approaches a limit - the goal of this study is to arrive at the lowest acceptable mesh density value, one producing results sufficiently close to the solution limit. This study is performed with a single nacelle position case - at height of 3.5 radii and $20 \%$ of the wing's chord.

The convergence limit of the data acquired is determined using a Richardson's extrapolation of the individually sampled data points. Because Richardson's extrapolation is most easily used with equally-spaced data points, the extrapolation is based on three solutions obtained at grid density levels of $25 \%, 50 \%$ and $100 \%$ of final density used.

\section{Table 2 - Solution Convergence}

\begin{tabular}{|c|c|c|c|}
\hline Density & $\mathrm{C}_{\mathrm{L}}$ & $\mathrm{C}_{\mathrm{D}}$ & $\mathrm{L} / \mathrm{D}$ \\
\hline $25 \%$ & 0.4 & 0.022802 & 17.542 \\
\hline $50 \%$ & 0.4 & 0.024356 & 16.423 \\
\hline $75 \%$ & 0.4 & 0.024862 & 16.089 \\
\hline $100 \%$ & 0.4 & 0.025075 & 15.952 \\
\hline $125 \%$ & 0.4 & 0.025071 & 15.955 \\
\hline
\end{tabular}

The observed order of convergence of the solutions is obtained using the equation [12].

$$
\begin{array}{r}
p=\ln \left(\frac{f_{3}-f_{2}}{f_{2}-f_{1}}\right) / \ln (r) \\
r-\text { grid refinement ratio }
\end{array}
$$

The extrapolation is then performed using the two finest grids using equation [13], and an estimate of the L/D ratio at zero grid spacing obtained.

$$
f_{h=0} \cong f_{2}+\frac{\left(f_{1}-f_{2}\right) r^{p}}{r^{p}-1}
$$

The Grid Convergence Index (GCI) is a measure of the percentage the computed value is away from the value of the asymptotic numerical value. The GCI is calculated using a safety factor of 1.25 according to the methodology provided in Roache ${ }^{10}$ and is listed alongside with the other results obtained in this study in Table 3 below. 
Table 3. Convergence Study Results

\begin{tabular}{|l|l|}
\hline Result & Value \\
\hline Order of Data Convergence & 1.249 \\
\hline Value of L/D at zero grid spacing & $\mathbf{1 5 . 9 6 3}$ \\
\hline Grid Convergence Index 1 (25\%-50\%) & $6.188 \%$ \\
\hline Grid Convergence Index 2 (50\%-100\%) & $\mathbf{2 . 5 0 3 \%}$ \\
\hline
\end{tabular}

The convergence of the solution, shown above to be sufficient by the GCI of $2.503 \%$ at the grid density used (100\% density) can also be observed in Figure 5 below.

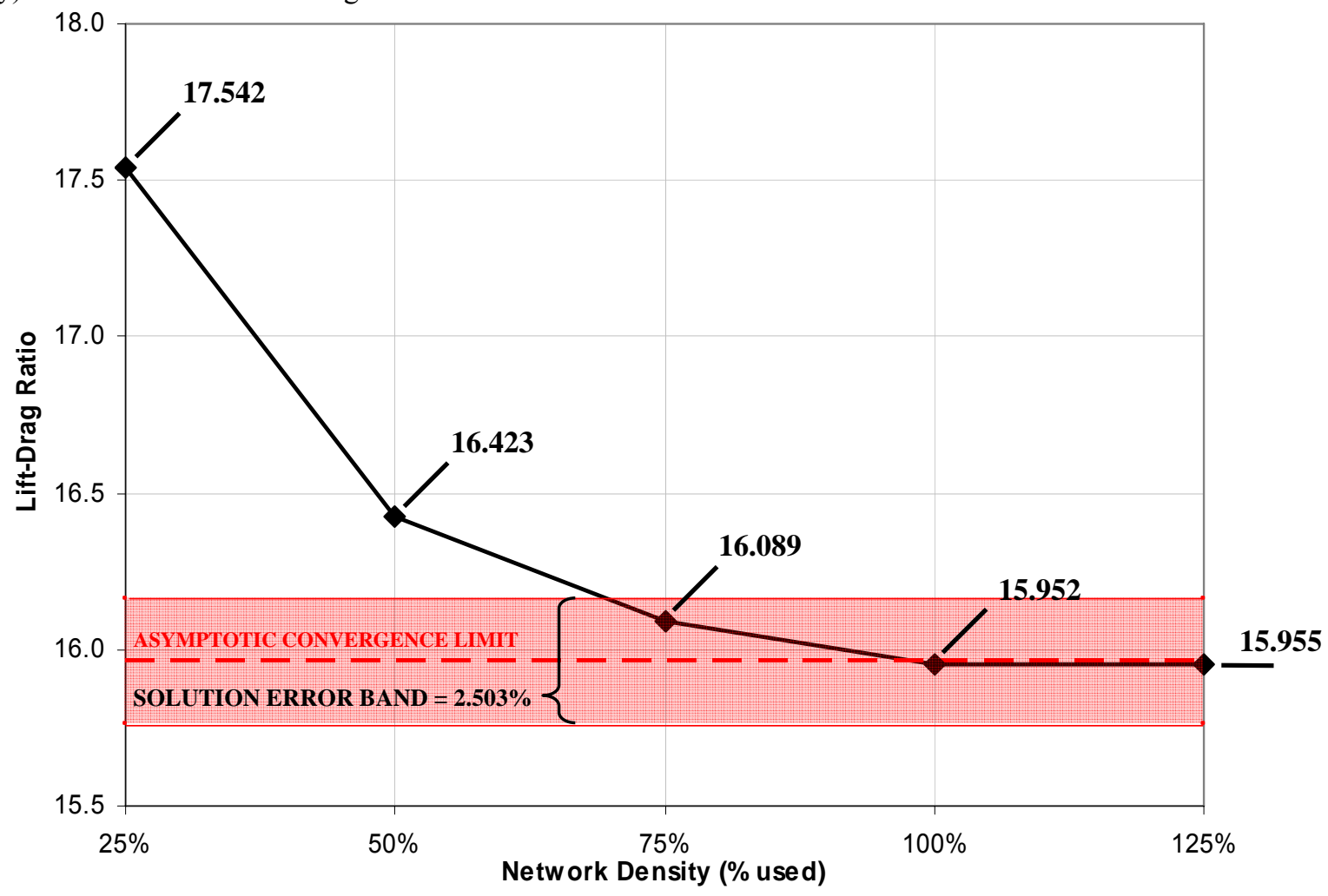

\section{B. Geometry Issues}

Figure 5. Grid independence study

The initial geometry used in the study contains a pylon, which connected the nacelle and the wing. This geometry took a significant amount of work to create, with the main issues being the surface trimming, gridding and network coincidence discussed earlier. The 3-component (wing, pylon, nacelle) can be seen below in Figure 6. 


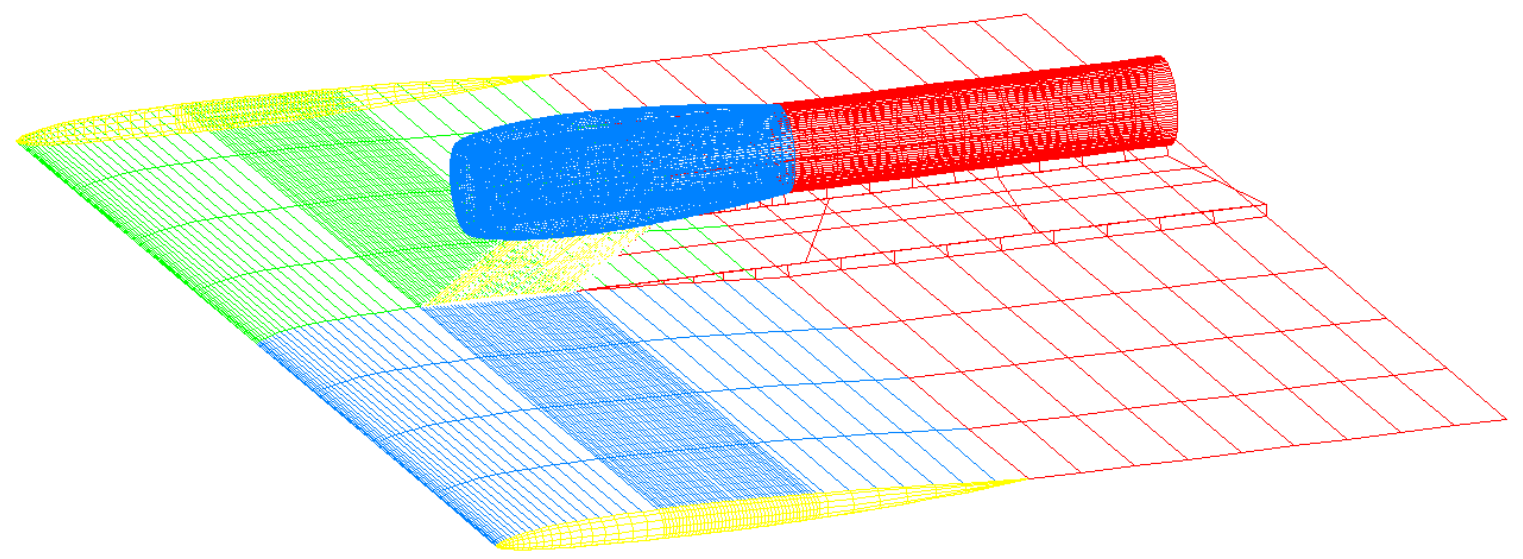

Figure 6. Generalized geometry (3-component version)

A number of issues result when this geometry is studied in TranAir. Although the general trends portrayed in the L/D curves generated are similar to those obtained with the two-component geometry, a number of anomalies make it difficult to have any confidence in the results. The results achieved with the two geometries compared at a single nacelle-height case (5.5 rad.) are compared in Figure 7 below.

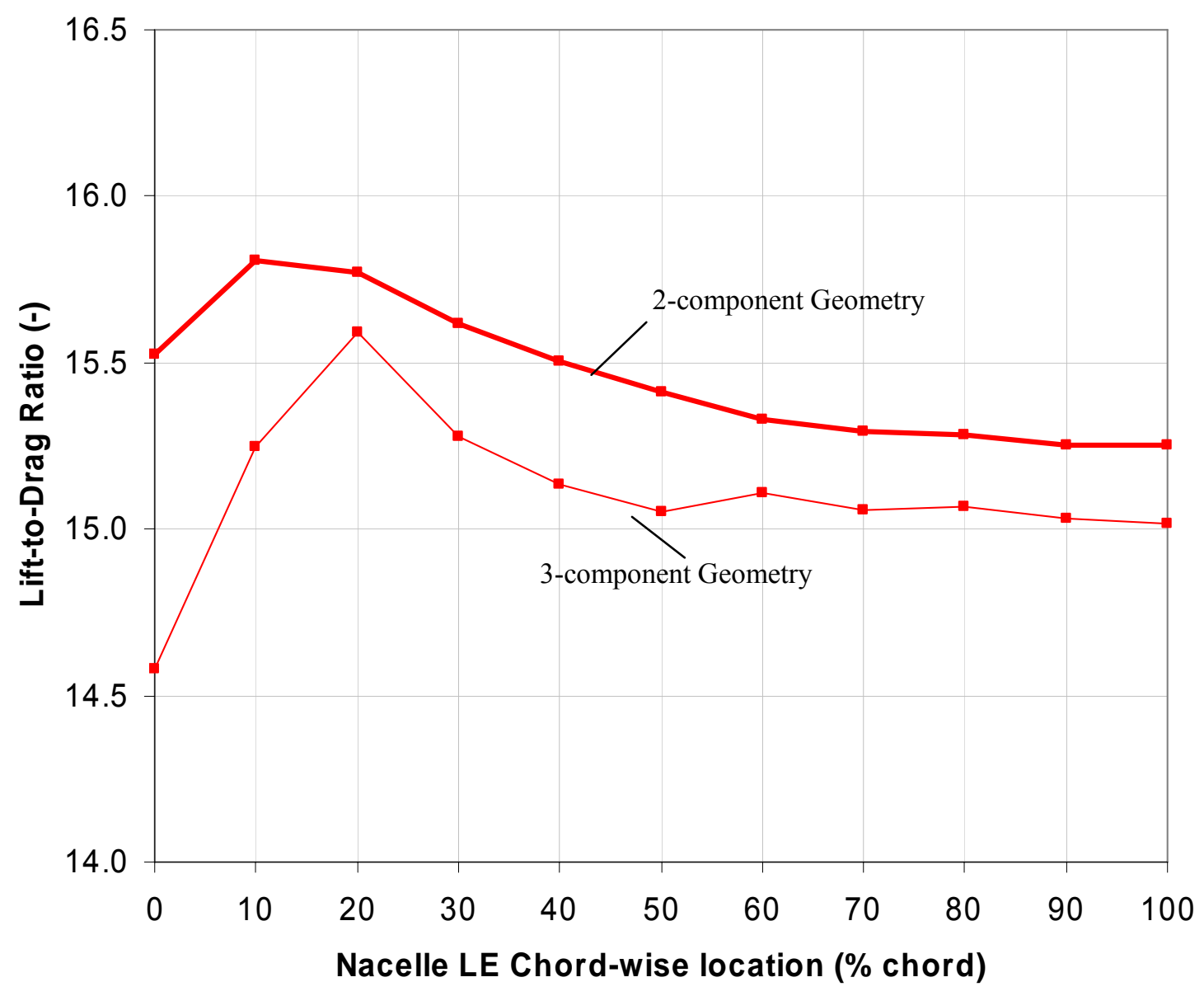

Figure 7. Comparison between 2-component and 3-component geometries

It can be seen that the drag curves are showing a similar trendline - they both have an L/D peak at approximately $20 \%$ of the wing's chord, and come to a gentle decrease as the nacelle nears the trailing edge of the wing. The two 
curves can be seen to be vertically spaced, a difference that can be attributed to the drag-generating influence of the pylon. It is, however, obvious that the trends obtained with the three-component geometry are less gradual and consistent. Even more specious results are encountered in other runs, while the two-component geometry produces consistently smooth results. Because the purpose of the study is to address the effects generated by the nacelle, and it appears that the pylon would only reduce the clarity of the results obtained, it was decided to remove the pylon from the study until a reason for this faulty operation could be found. Another benefit of reverting to the pylon-less geometry is that the gridding on the nacelle and the wing can now be kept constant across the range of geometries used in this study, instead of having zones of denser gridding corresponding to that used on the pylon. This allowed for much faster model generation, as the same boundary layer definitions could be used across all runs.

\section{CFD Results}

After performing CFD analysis on the established range of nacelle locations, values of Lift-to-Drag ratio for the wing-nacelle-pylon assembly were obtained. The plot showing the relationship of lift-to-drag to nacelle location can be seen in Figure 8.

$\mathrm{L} / \mathrm{D}$

Ratio

$(-)$

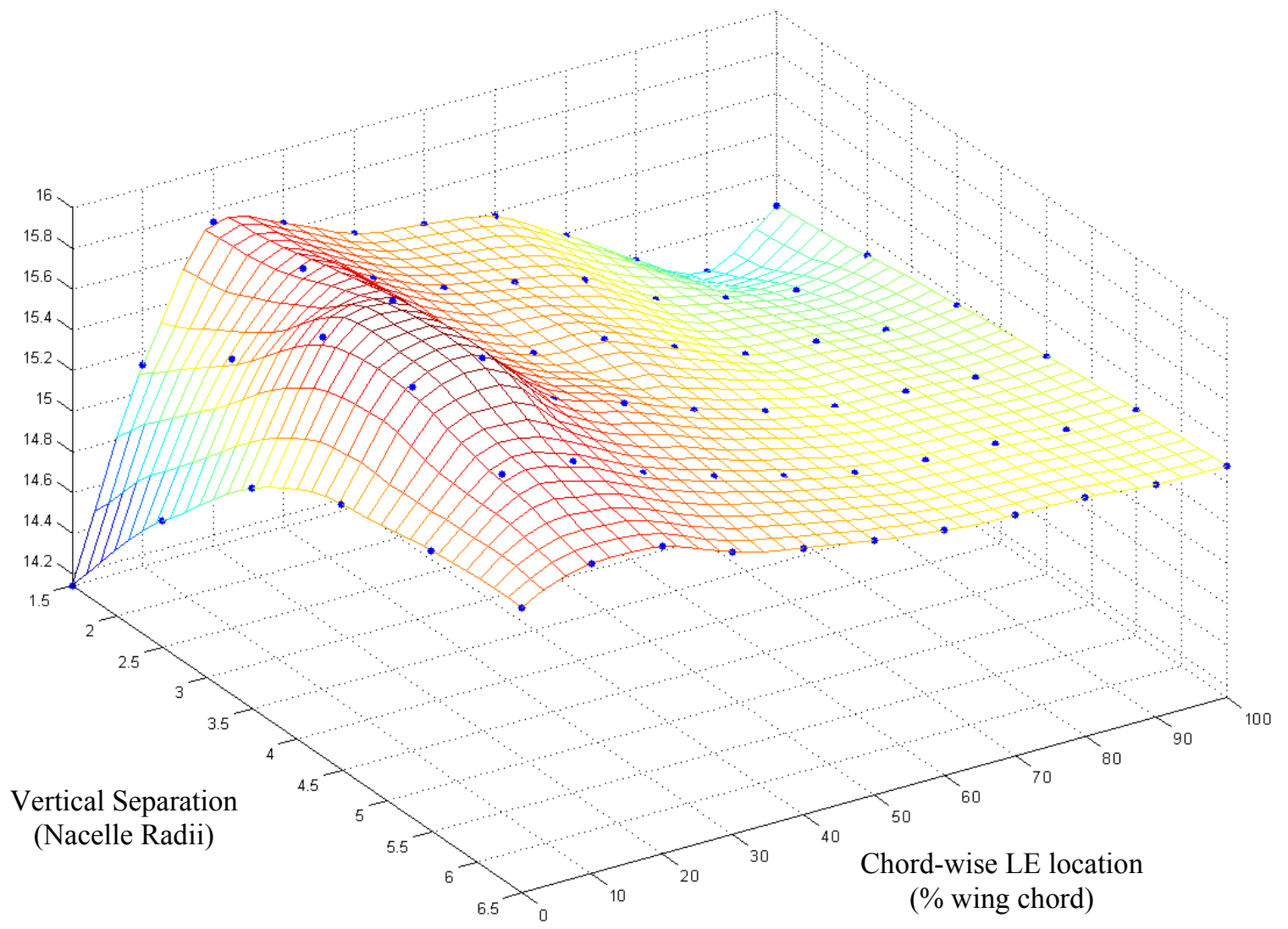

Figure 8. Surface Map of CFD Results

A number of general trends can be noticed in the plot. Irrespective of nacelle mounting height, the L/D peak appears to correspond to an intake leading edge location at approximately $20 \%$ of wing chord. A 2-dimensional view of the $\mathrm{L} / \mathrm{D}$ curves can be seen in Figure 9 below. Although the 3-dimensional L/D surface is more difficult to visualize in this view, the plot is much more useful for datapoint-acquisition purposes. 


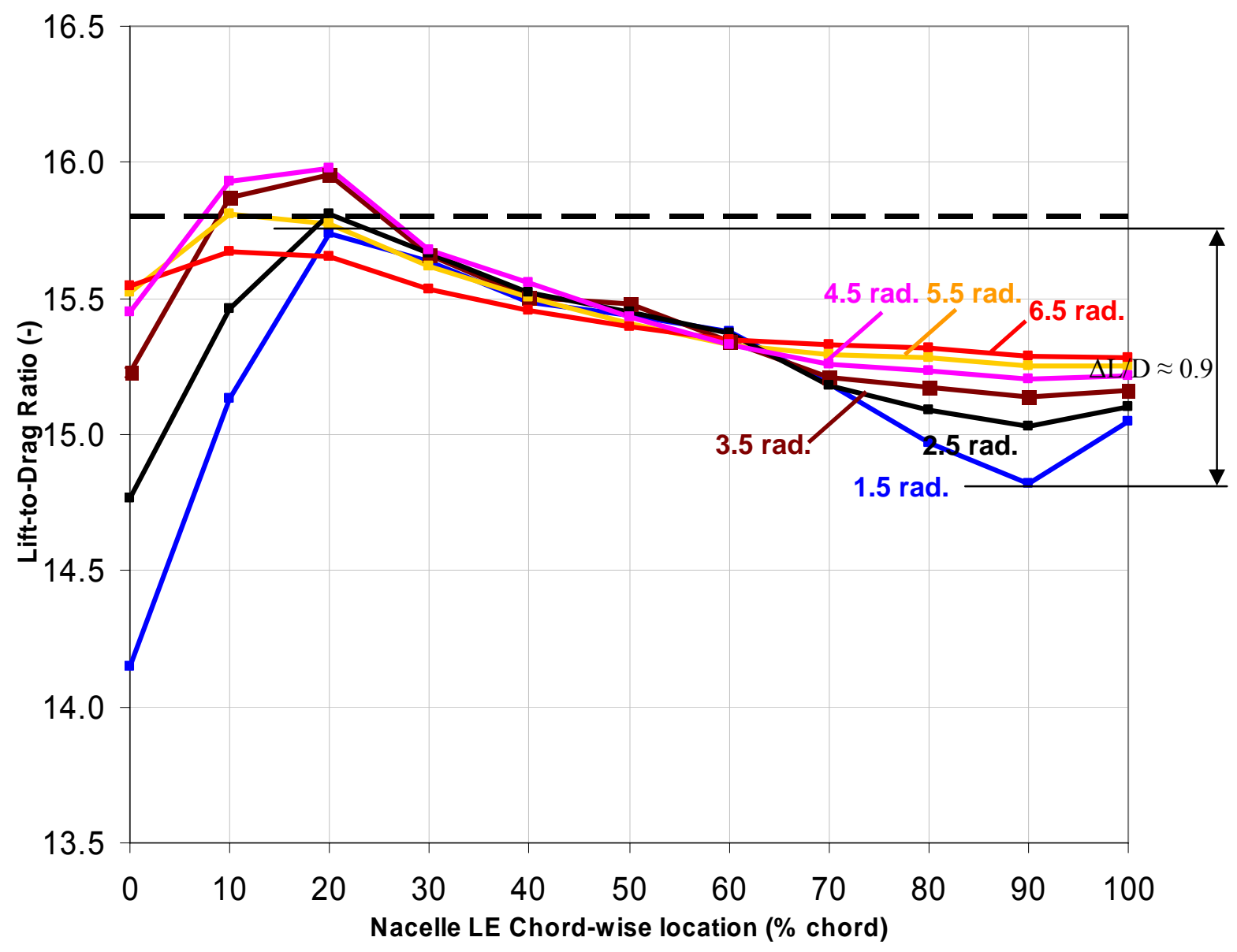

Figure 9. 2-d Projection of CFD Results Map

The dashed horizontal line on the plot above indicates the L/D ratio of the wing tested alone, without the nacelle, at the prescribed $\mathrm{C}_{\mathrm{L}}$ value. A number of the $\mathrm{L} / \mathrm{D}$ points of the tested wing+nacelle geometries can be seen to actually exceed the lift-generating efficiency of the clean wing, even with the addition of the nacelle. By careful positioning of the nacelle it is therefore possible to enhance the performance of the clean wing.

In the process of moving the nacelle chordwise between the two extremes at the leading and trailing edges of the wing, the L/D ratio of the entire installation can be seen to be significantly affected. The largest L/D ratio change caused by chord-wise movement of the nacelle, observed at a vertical separation of 1.5 radii is approximately 0.9 . The L/D ratio change is the highest at this low separation, due to the high drag generated in the near-leading-edge nacelle locations, but the global peak L/D ratio is achieved at a higher vertical separation case.

A trend can be seen in the effects of nacelle vertical separation, defined in nacelle radii between axis of nacelle and wing chord line. The L/D ratio can be seen to peak significantly later with respect to this parameter, with the overall $\mathrm{L} / \mathrm{D}$ peak corresponding to a nacelle axis vertical separation of 4.156 nacelle radii. With further vertical separation, the L/D ratio can be seen to smoothly decrease. From detailed flow visualizations addressed later in this study it is likely that this is caused by the diminishing of nacelle influence on the flow over the wing upper surface.

In order to better understand the trends portrayed in the L/D ratio surface, it will be useful to first analyze the local flow behaviors seen in two geometry cases differing in nacelle-wing surface separation.

The first case (50\% chord, 1.75 radii), shown in Figure 10 displays a number of interesting flow characteristics. Most importantly, a zone of low-speed flow can be seen directly ahead of the intake face. This zone is generated by the upstream influence of the nacelle body, and can be seen to decrease the speed of flow that would normally be seen at that point on the wing. 


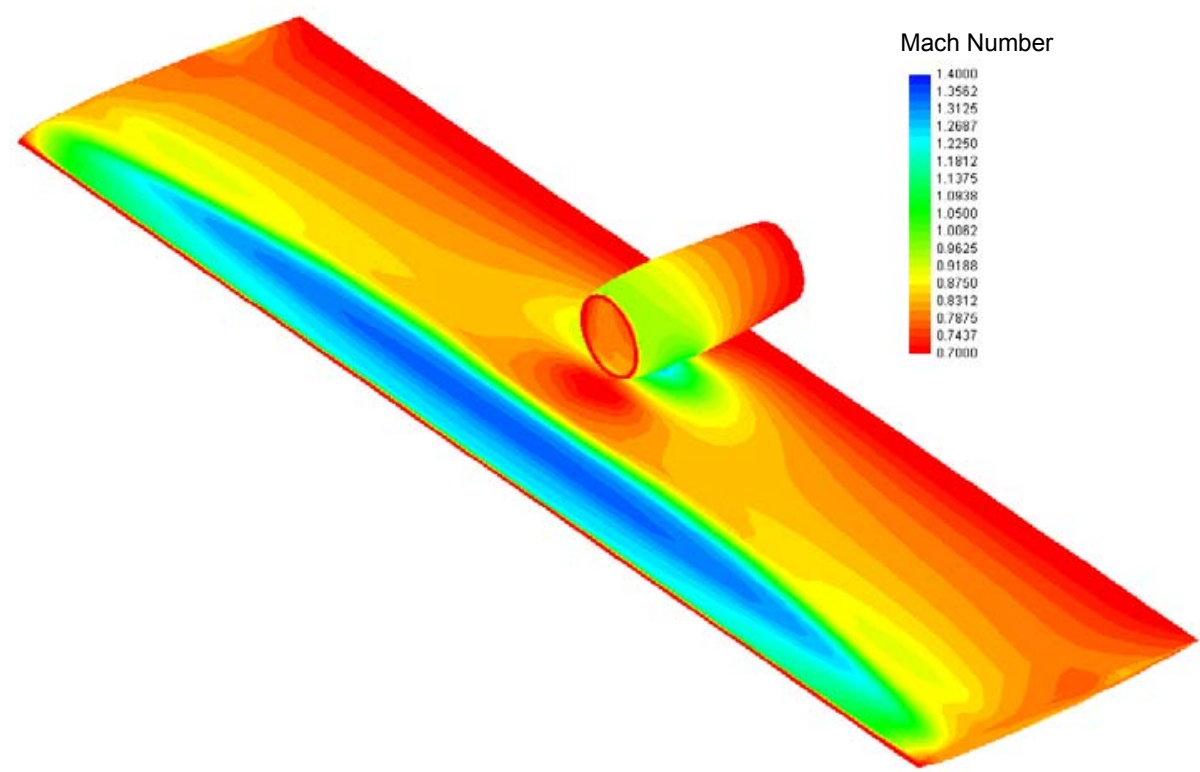

Figure 10. Nacelle at $50 \%$ wing chord, 1.75 radii, $C_{L}=0.4$

However, a second, less beneficial behavior can also be observed in the figure. A local zone of high-speed flow can be seen at the nacelle-wing interface. The flow can be seen to accelerate to approximately Mach 1.3 within the convergent-divergent channel formed by this interface. This flow, and the shock associated with it produces various undesirable flow phenomena, which in the end amount to increasing the wing drag.

The second case (50\% chord, 6.75 radii) shown in Figure 11 has a much higher degree of nacelle separation than that shown in the first case.

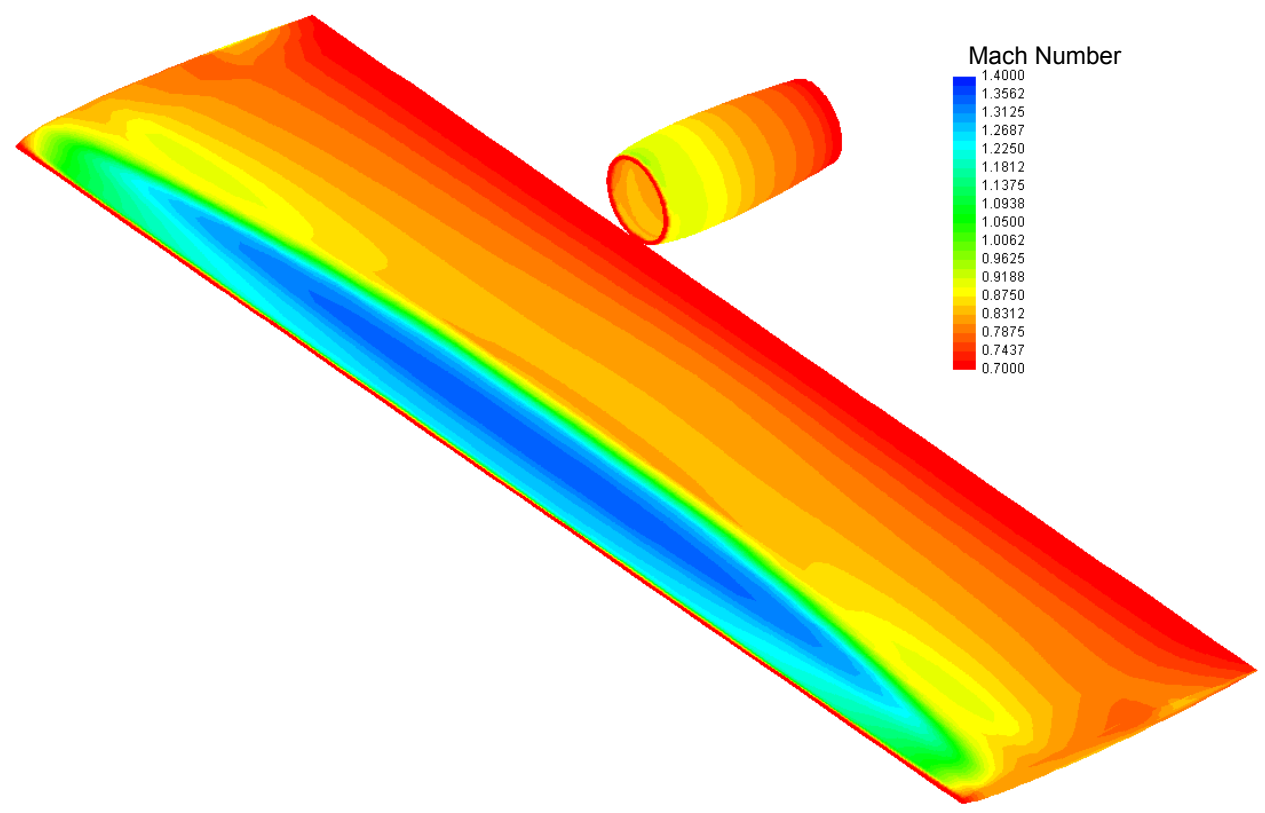

Figure 11. Nacelle at $50 \%$ wing chord, 6.75 radii, $C_{L}=0.4$

The maximum local Mach number area is visible at the suction peak of the wing. Because of the large separation between the nacelle and the wing surface, no appreciable compression occurs in the wing-nacelle channel and the upper surface of the wing shows no signs of the influence of the nacelle. 
It is also useful to evaluate the changes brought on by modifying the chord-wise location of the nacelle, while leaving the vertical separation constant. By taking a section through the geometry at a span-wise location coincident with the centerline of the nacelle, characteristics of the airflow around the geometry can be better visualized. In Figure 12 below, the initial effects of nacelle suppressing high-speed flow on the top surface of the wing can be seen.
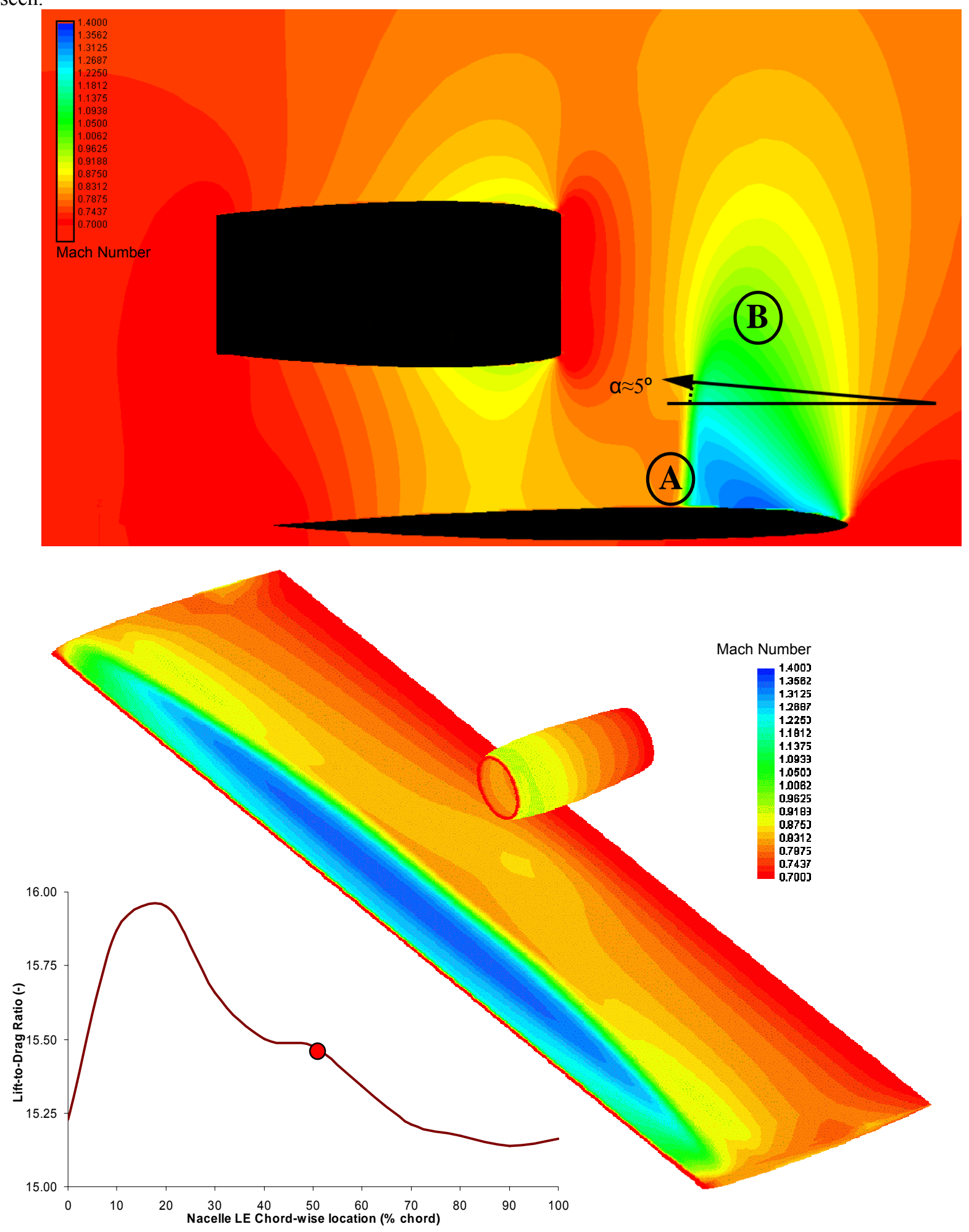

Figure 12. Nacelle at $50 \%$ wing chord, 3.5 radii, $C_{L}=0.4$ 
The first flow example discussed shows little to no nacelle influence on the flow on top of the main wing. A flow behavior of particular interest is the shock that can be seen to occur in zone ' $A$ ', with the flow rapidly decreasing speed from Mach 1.4 to freestream speed of Mach 0.75. This is likely the cause of the reduced L/D ratio shown by this wing geometry, as the large area of shock significantly impairs the wing's aerodynamic performance. Also, the zone of high-speed Mach 1.4 flow can be noticed to be quite large, progressing approximately $1 / 4^{\text {th }}$ of the way along the chord of the wing until shocking down to subsonic speed. The vertical influence of the leading edge suction zone is quite significant, with the supersonic flow area, demarked by 'B', extending approximately one half chord length above the wing surface.

The flow behavior which results when a nacelle is positioned further forward to a location $20 \%$ behind the wing's leading edge can be seen in Figure 14, and is discussed in detail in the following paragraphs.

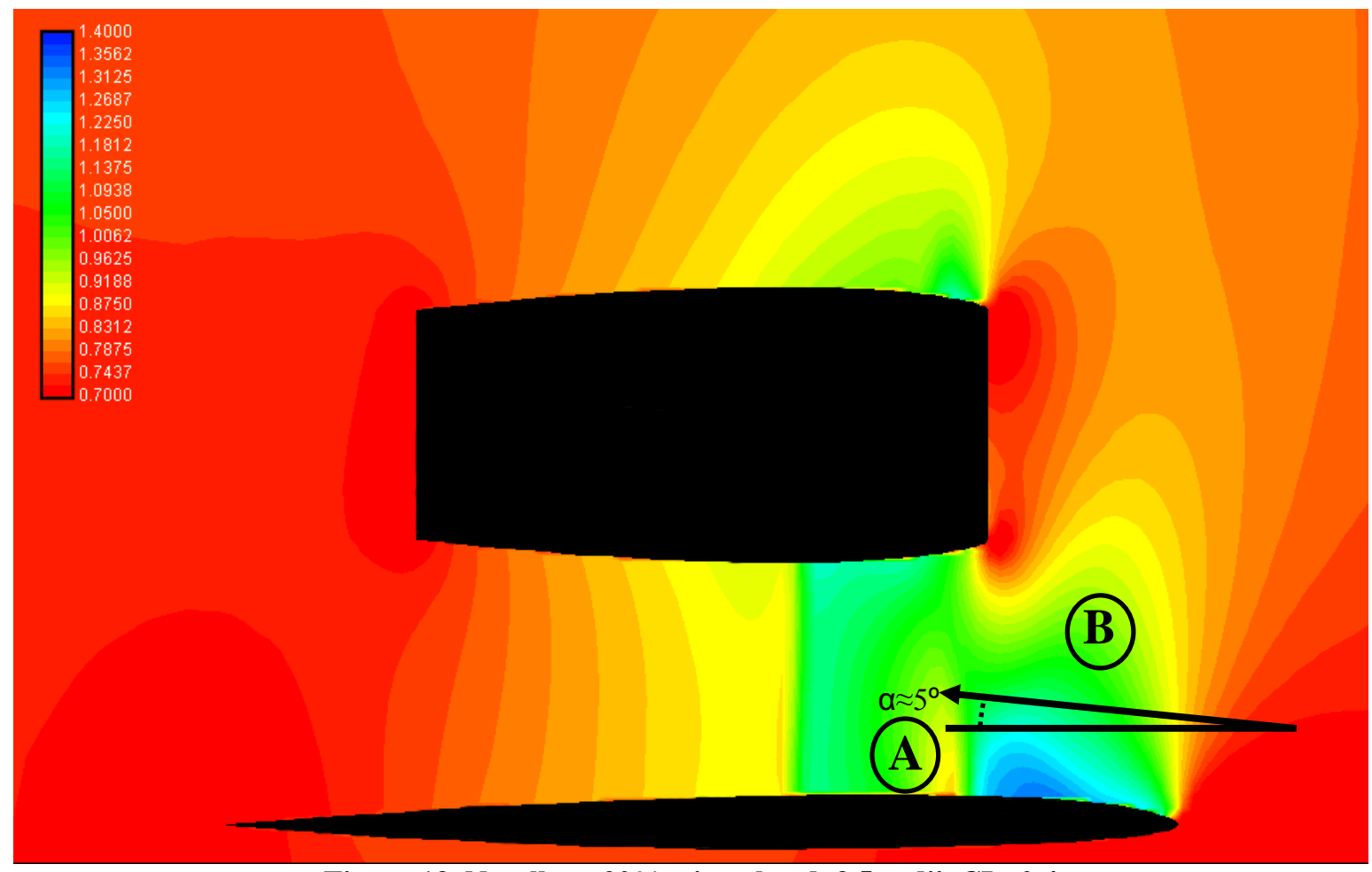

Figure 13. Nacelle at $20 \%$ wing chord, 3.5 radii, $\mathrm{CL}=0.4$ 


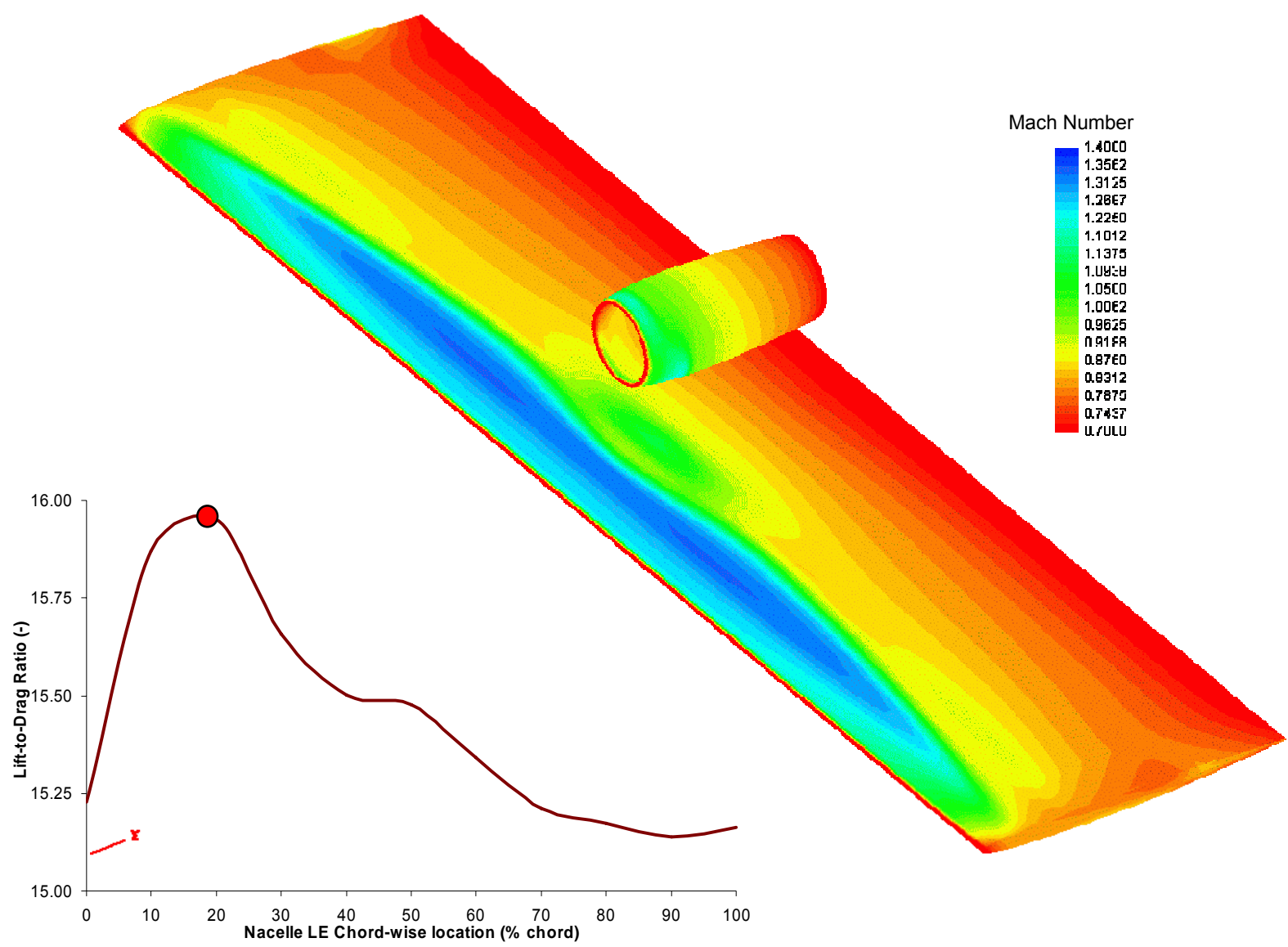

Figure 14. Nacelle at $20 \%$ wing chord, 3.5 radii, $C_{L}=0.4$

The L/D ratio of the configuration can be seen to increase after the nacelle is introduced in a location closer to the leading edge suction zone. The flow in the above picture illustrates a reduction in the amount of supersonic shock-down present in front of the nacelle leading edge. A small shock, demarked by ' $A$ ', can be seen very close to the surface of the wing, followed by a zone of subsonic flow that is again rapidly accelerated. However, the main quantity of high-speed flow progresses further, undergoing shock-down to sonic speed only after it has passed approximately $1 / 3^{\text {rd }}$ of the way down the nacelle. The main normal shock that occurs appears to be a much weaker than the one in the previous case, with the majority of the high-speed flow having a sonic number below Mach 1.1. The presence of the nacelle body acts to decrease the magnitude of the shock-down that occurs behind the suction zone.

The span-wise extent of the influence the nacelle body has on the flow field is surprisingly large, considering the comparatively small diameter of the nacelle. This can best be seen in the isometric view of the geometry, which shows the increased sonic zone around the front $1 / 3^{\text {rd }}$ of the nacelle, and the reduced high-speed flow zone around the leading edge suction peak. Also of interest is the decrease in the size of zone ' $\mathrm{B}$ ', the separated zone of highspeed flow. Being suppressed by the low-speed front generated ahead of the nacelle, supersonic flow can be seen to occupy a smaller area above the wing suction zone.

In order to better evaluate the effect of the nacelle flowfield on wing performance, it is helpful to analyze the surface pressure coefficients on the top and bottom wing surfaces. These, displayed at a number of span-wise sections taken through two versions of the geometry are presented in Figure 15 and Figure 16 below. 

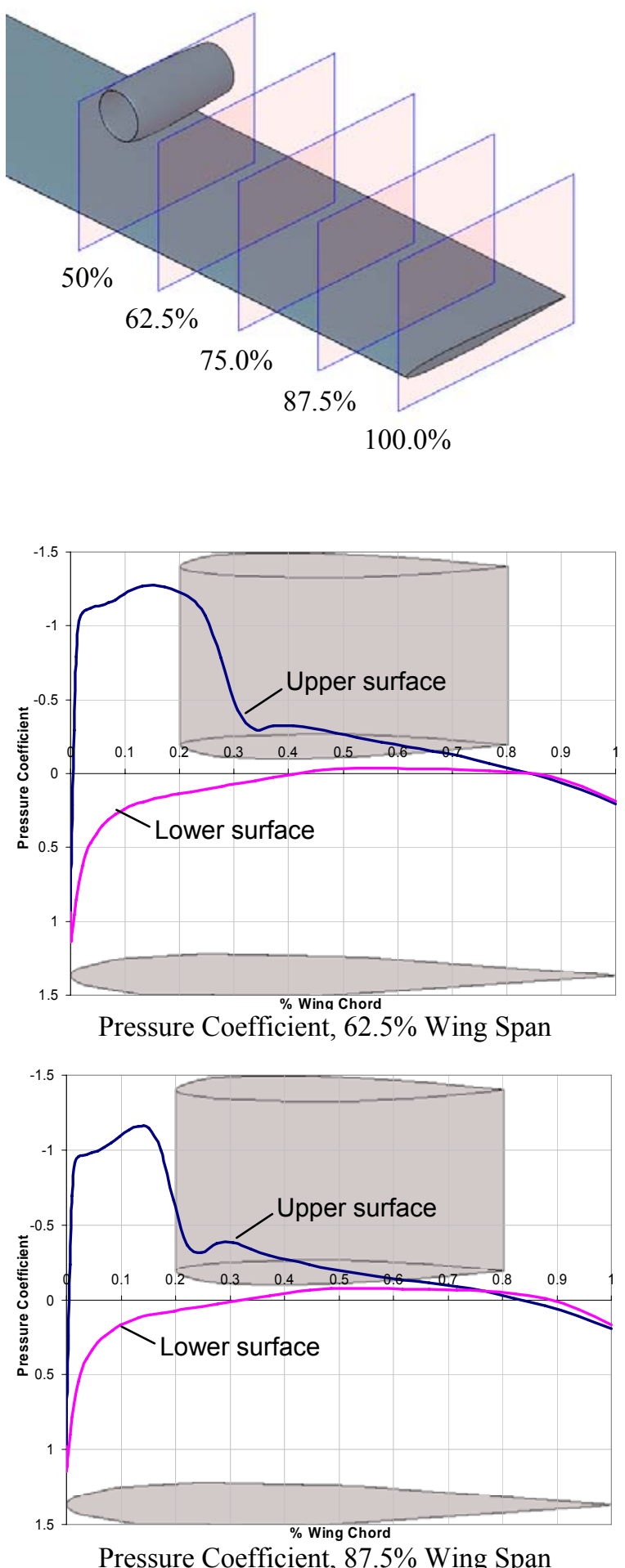

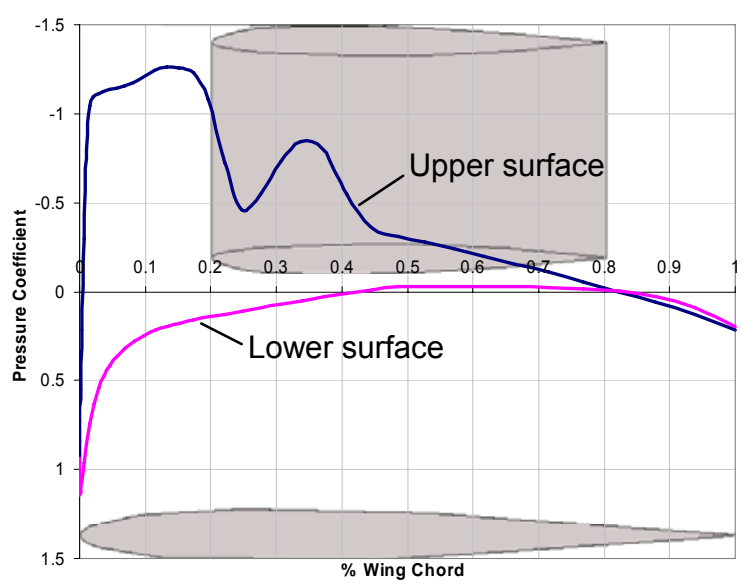

Pressure Coefficient, 50\% Wing Span

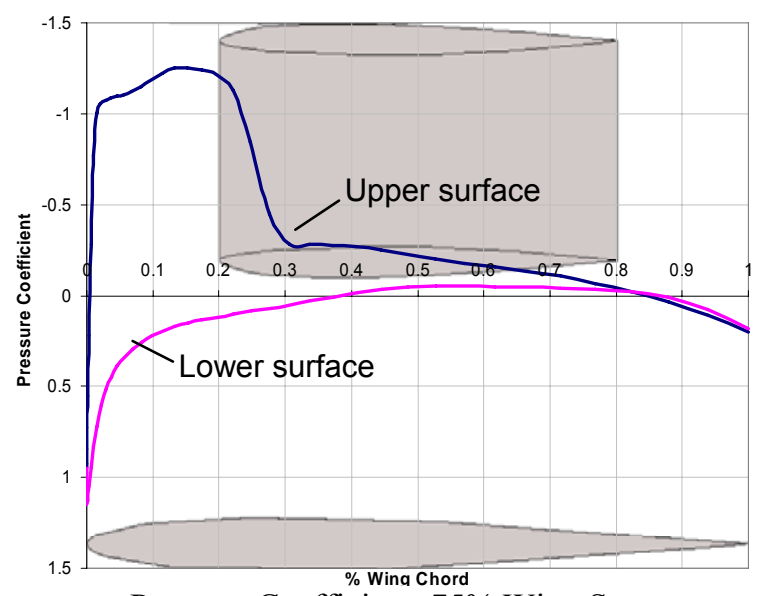

Pressure Coefficient, 75\% Wing Span

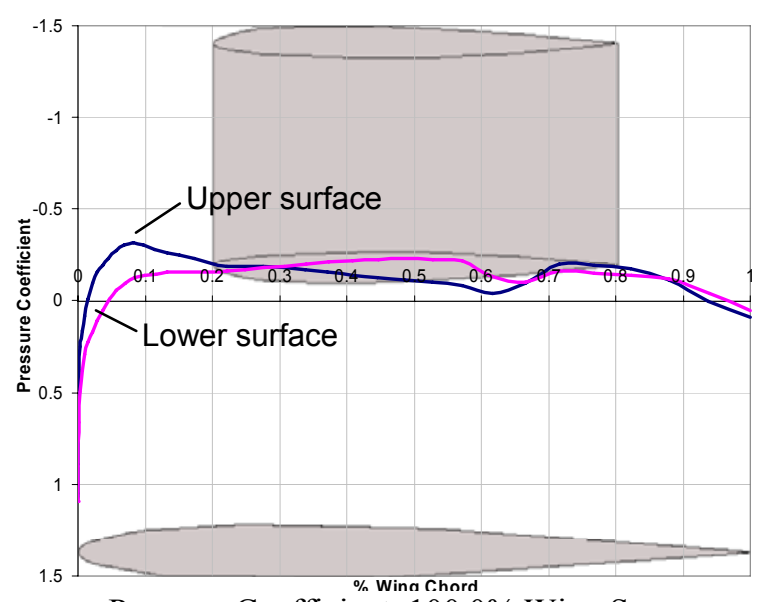

Pressure Coefficient, $100.0 \%$ Wing Span

Figure 15. Upper and Lower Surface Pressure Coefficients, Nacelle Present 

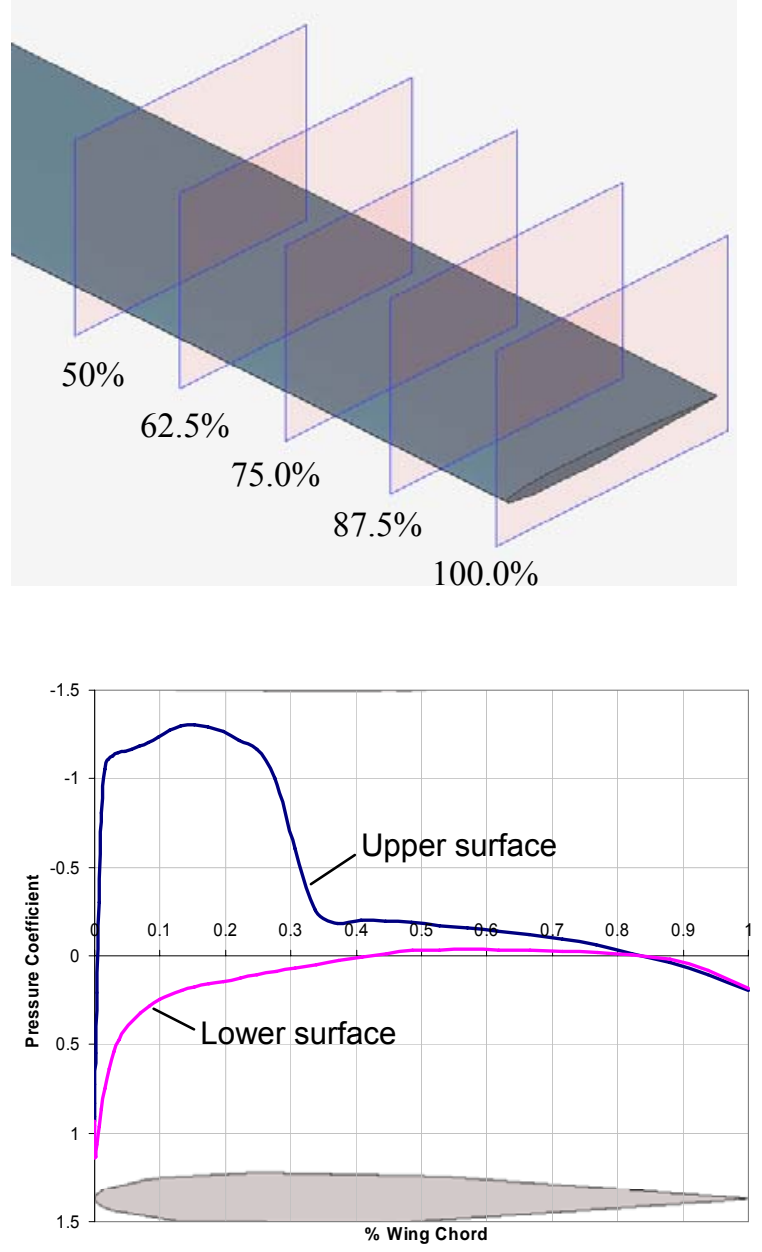

Pressure Coefficient, $62.5 \%$ Wing Span

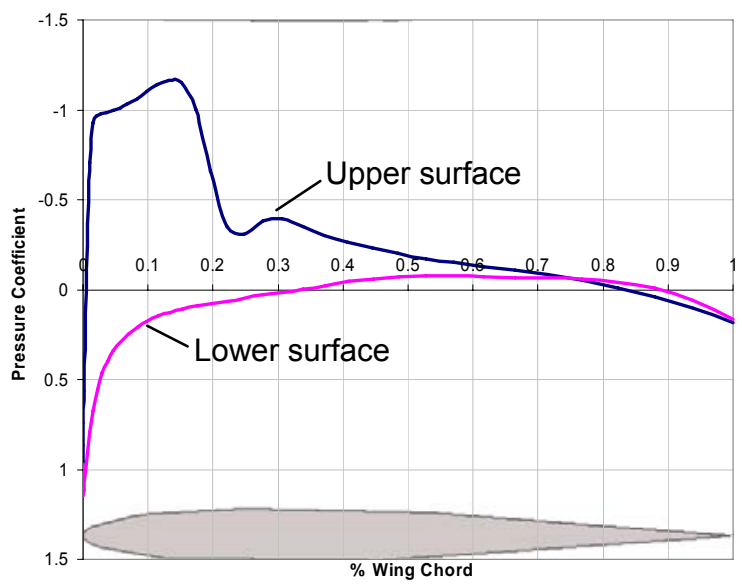

Pressure Coefficient, $87.5 \%$ Wing Span

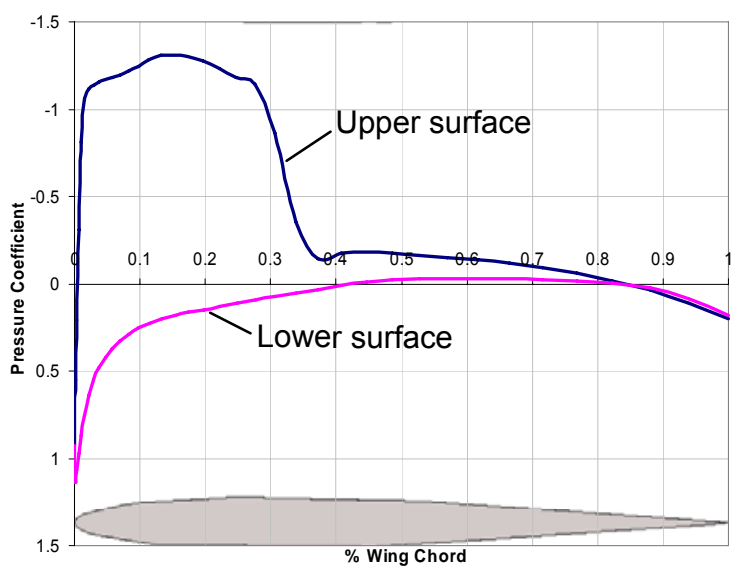

Pressure Coefficient, 50\% Wing Span

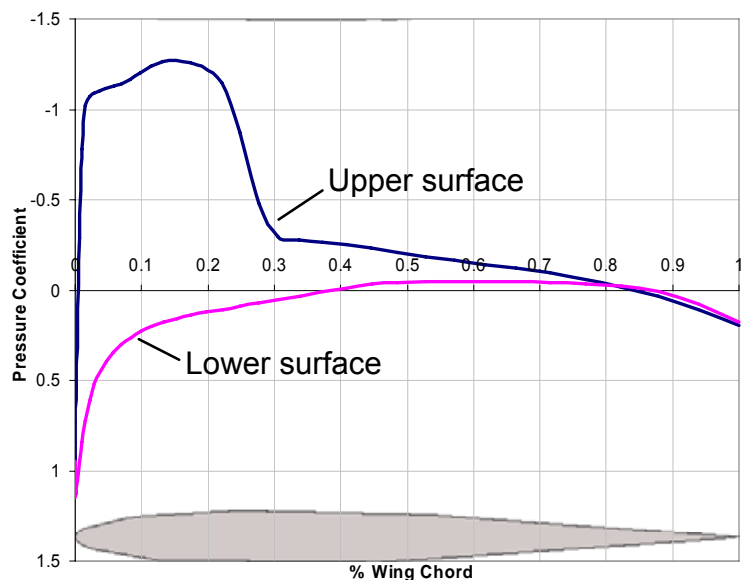

Pressure Coefficient, 75\% Wing Span

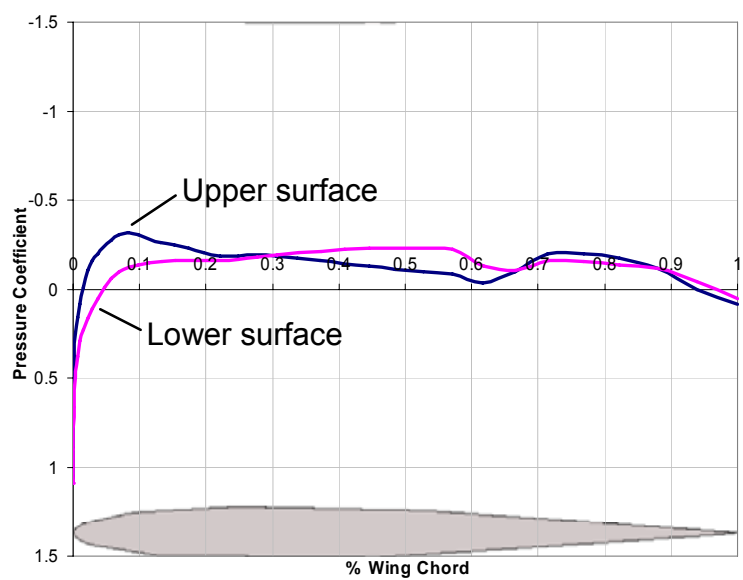

Pressure Coefficient, $100.0 \%$ Wing Span

Figure 16. Upper and Lower Surface Pressure Coefficients, No Nacelle

The pressure drop present behind the supersonic shock at approximately $30 \%$ wing chord is visible in all section cuts except the one at $100 \%$ wingspan, where wingtip effects are most prevalent. A comparison of the two pressure contour cases at $50 \%$ wing span shows the primary effect of the nacelle body on upper surface pressure coefficients, 
and as a result, on wing lift capability. While showing the same initial pressure drop as in the other section cuts, the pressure coefficient again increases between approximately $25 \%$ to $45 \%$ wing chord. This effect, discussed earlier in terms of airflow Mach number underneath the nacelle shows the drag reducing capability the presence of the nacelle body affords to the wing.

It is useful to compare the normalized spanwise lift distribution plots of the $20 \%$ wing chord 4.5 radii separation case and that of a clean wing with no nacelle present. A normalized parabolic lift distribution present on a clean wing is shown in Figure 17 below.

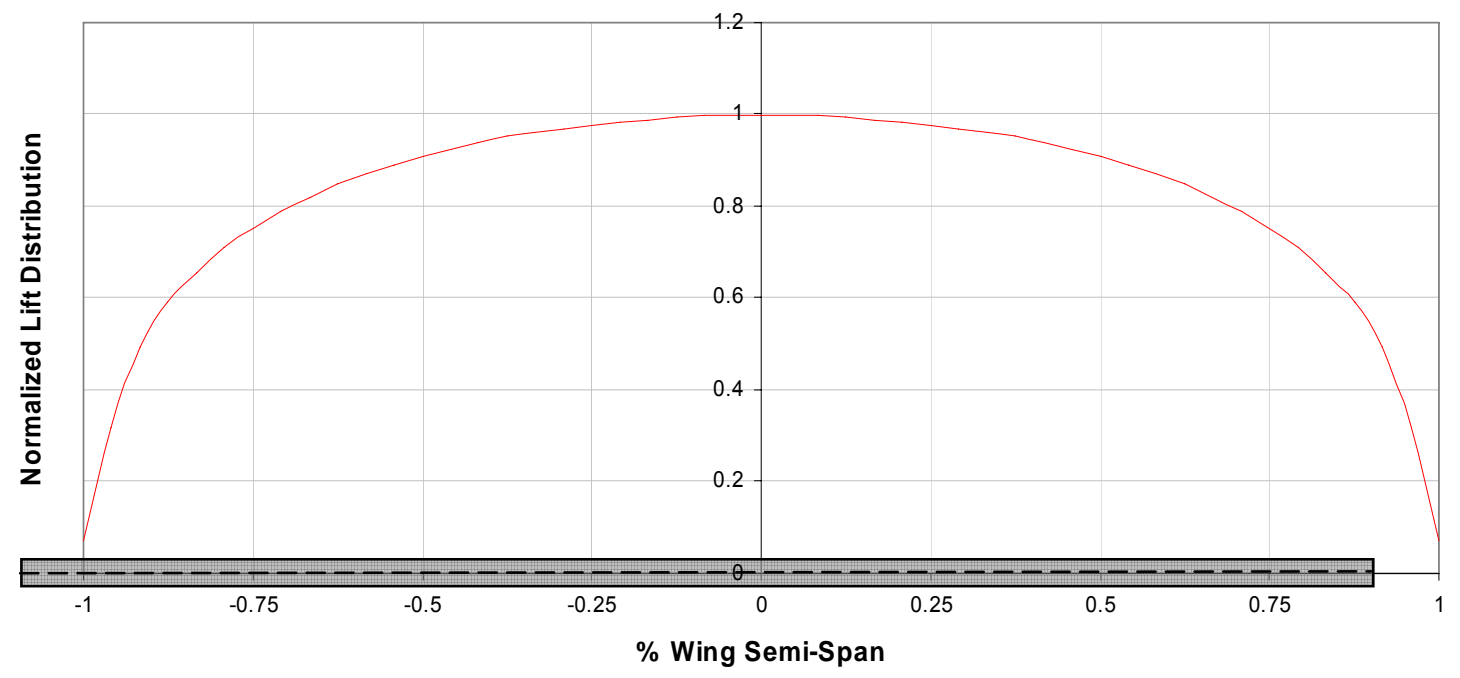

Figure 17. No-nacelle Spanwise Lift Distribution

After conducting a comparison, it was observed that the lift distribution of the nacelle-on case is only slightly altered by the nacelle's presence. To clearly compare the two curves without the overlap which tends to occur at the $y$-axis scale used in the previous figure, the difference between the nacelle-on lift distribution and the clean wing lift distribution is presented in Figure 18.

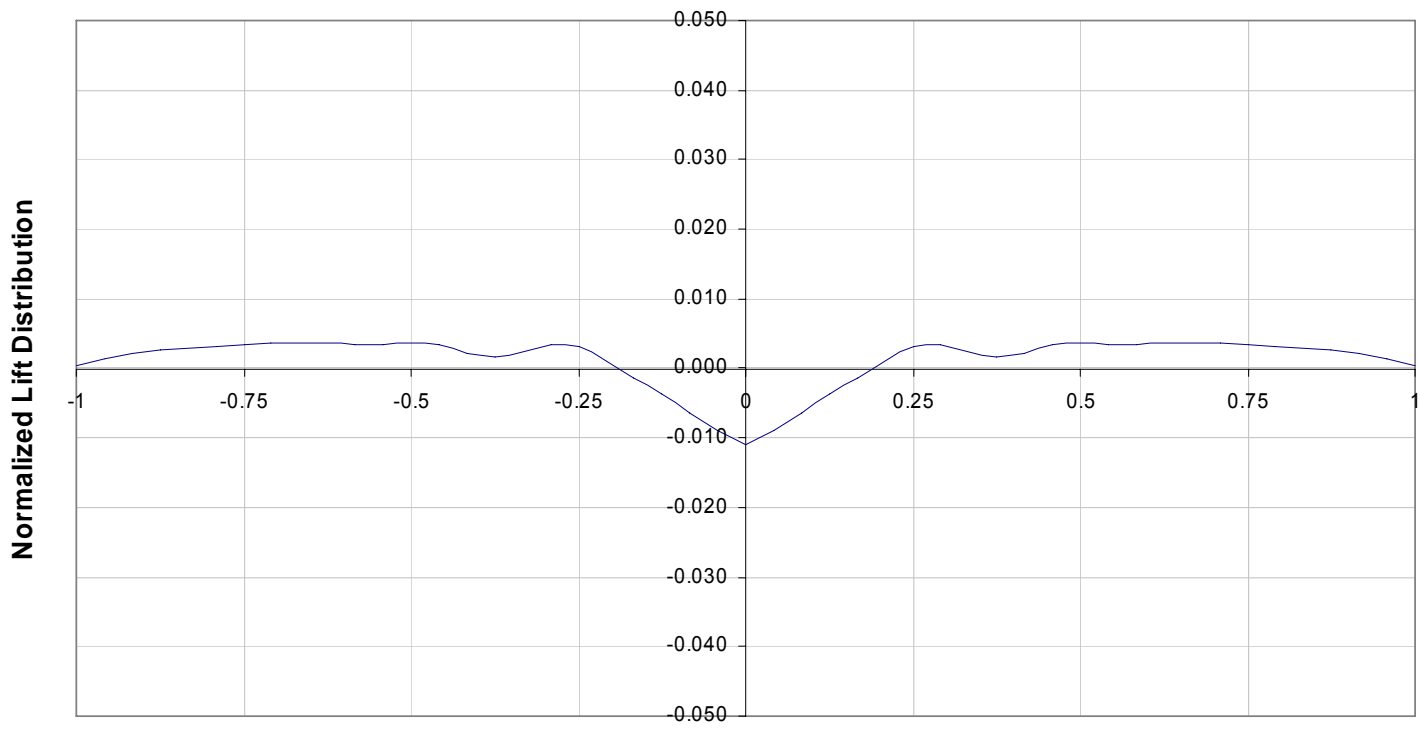

$\%$ Wing Semi-Span

Figure 18. Spanwise Lift Distribution Difference

It should be noted that in order to achieve equal values of lift on the two wings being compared, the $\mathrm{C}_{\mathrm{L}}$ goal of 0.4 is set for the networks of the wing, while in the rest of the study the $\mathrm{C}_{\mathrm{L}}$ goal is defined for the entire wing + nacelle geometry. As can be seen from the above plot the sectional lift is slightly affected by the nacelle presence (at most 
by $1 \%$ at the root). The wing's normalized lift distribution is still of a parabolic shape, but with a slight flattening present below the nacelle and an area of higher lift located outboard of approximately $25 \%$ semispan. With the areas under their respective lift distribution curves being equal, both of the wing cases produce the same amount of lift. The increase in the assembly's L/D ratio is achieved via a reduction in drag resultant from the lower flow Mach number at the suction zone and a weaker shock.

In order to conduct the study at a single lift coefficient, TranAir CFD code automatically modified the angle of attack to achieve a goal $\mathrm{C}_{\mathrm{L}}$ of 0.4 for all geometry cases evaluated. The effects of both the nacelle chordwise and vertical location on the angle of attack necessary to achieve the required $C_{L}$ can be seen in Figure 19 below.

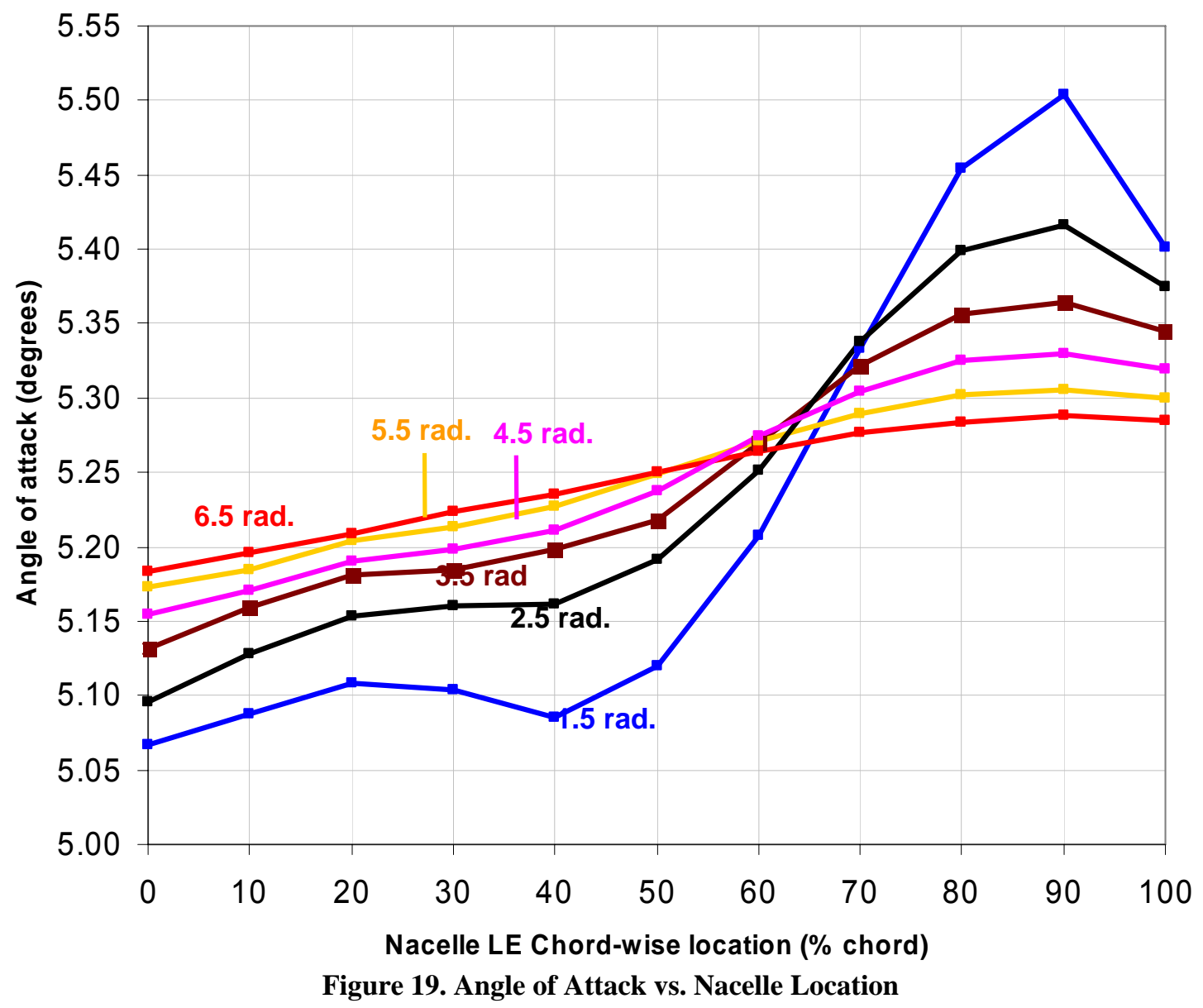

The cases with the lowest vertical separation have the highest variance of angle of attack as a result of the stronger effect the nacelle flow field has on the upper surface of the wing. The general trend that can be observed is that of the angle of attack growing with the movement of the nacelle towards the trailing edge of the wing. This trend is most clearly observed at the higher vertical-separation cases, where it is not obscured by local flow phenomena present when the wing and nacelle are in close proximity. Configurations with the nacelle close to the leading edge of the wing achieve a greater level of high-speed flow suppression in the vicinity of the leading-edge suction zone. The resultant lower pressure in the suction zone allows the wing to create lift at a lower angle of attack. As the nacelle nears the trailing edge, the reduction in its effectiveness in suppressing high-speed flow results in higher pressures in the suction zone, forcing the wing to a higher angle of attack to achieve a given $C_{L}$ value.

An important point that requires discussion is the fact that the aerodynamic results obtained in this study appear to be very different from those claimed in the patent which prompted this study. The nacelle mounting range claimed to exhibit drag reduction and drag divergence Mach number increase in the patent is between $60 \%$ and $80 \%$ of wing chord. Because this study was conducted at a single Mach number, the drag divergence claims of the patent could not be verified, but obviously, the area of drag reduction that is seen in this set of results is significantly 
different from that in the patent. It is very likely that an aspect of the study which could not be replicated caused this discrepancy.

The wing airfoil used in the original study is proprietary to the Honda Corporation and therefore could not be used. No performance data is currently available on this airfoil, but a schematic obtained from the patent and presented in Figure 20 shows the wing generating a supersonic shock at an approximately $70 \%$ chord location.

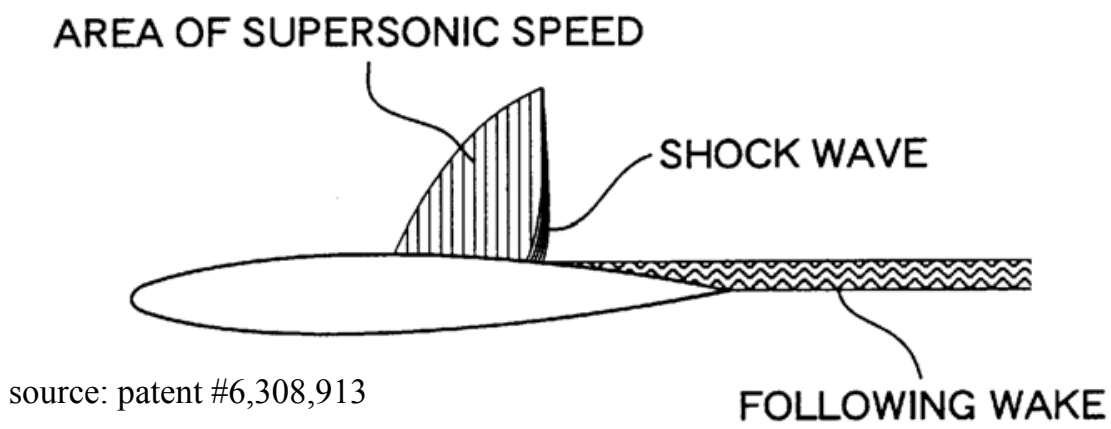

Figure 20. Honda Airfoil Schematic

Although transonic or "supercritical" airfoils should produce a suction peak much further down the airfoil than the conventional $25 \%$ location, the observed edge of the suction and supersonic flow zones on the Gulfstream airfoil used reached approximately $30 \%$ from the leading edge. Obviously, this shock location is significantly different from that present on the wing used in the patent. As shown earlier, the main drag-reducing effect of nacelle is obtained from aerodynamic interaction with the wing's upper-surface shock. It is therefore likely that the location of the shock will define the optimum location of the nacelle, and a different, better performing airfoil would likely go a long way toward explaining the differences seen between the results of the two studies.

Establishing an ideal nacelle location is accomplished by suppression of local supersonic flow at the leading edge suction zone, and the reduction of flow speed at the wing-nacelle interface. However, the weight of structure required to achieve the best location from the aerodynamic standpoint is another aspect of this compromise that must be considered. Finally, the relative value of these factors varies with the mission that the aircraft with this engine installation is designed for. For example, a long-range aircraft will be able to garner more benefit from a lowdrag installation, while a short-range aircraft is likely to benefit more from a higher-drag but lower-weight installation. To achieve a balance of all these aspects is the main goal of this study; it was addressed using MultiDisciplinary Optimization.

\section{MDO Results}

After an iterative optimization and analysis of aircraft performance, the optimum nacelle location was found at $15.9 \%$ wing chord, and with a separation of 4.0 radii. Although the most optimal position from an aerodynamic standpoint occurs at a nacelle location of $15.9 \%$ wing chord and a separation of 4.156 radii, the structural constraint forces the optimum overall location to a point slightly closer to the wing surface. The final nacelle location is illustrated in Figure 21 below. 


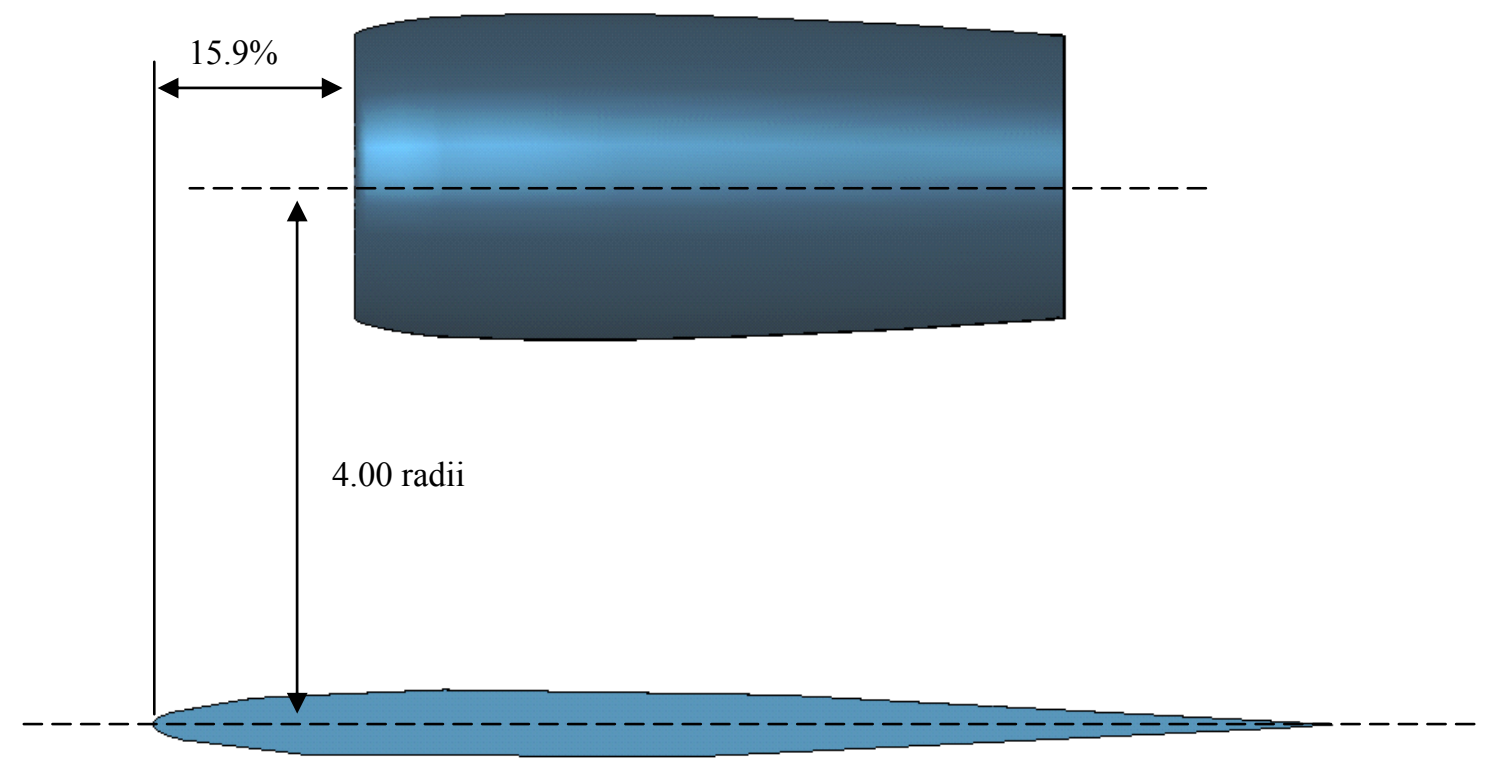

Figure 21. Optimum Nacelle Position at $15.9 \%$ chord, 4.0 radii.

It is difficult to compare the overall efficiency results obtained in this section with those that could be expected from an aircraft with a conventional engine mounting scheme. However, it is possible to show the beneficial effects of mounting location optimization using the MDO study. The locations evaluated within the MDO study range ranged from Nacelle LE from $0 \%$ to $100 \%$ wing chord, and Nacelle Axis from 1.5 to 5.5 radii separation from wing chord line. In this range, the most optimal location allows for an aircraft that carries $160 \mathrm{lb}$ more payload than the least optimum location, equating to a $4.5 \%$ increase in payload capacity. If the same aircraft has an option of using the excess payload capacity in form of fuel, it can extend its mission cruise range by 155 nautical miles, a $6.2 \%$ increase.

\section{Conclusion}

In the course of this study, a complete set of tools to develop and analyze wing and nacelle geometries in Boeing TranAir was created, with an MDO tool used to analyze the effects as applied to a notional aircraft. The results obtained in the aerodynamic part of the study, although significantly different from those described in US Patent $\# 6,308,913$, do appear to show a number of important trends. A variety of important lessons in operating Boeing TranAir and creating geometry using Boeing AGPS were learned, with this information complied and presented in detail in the full paper describing this study ${ }^{11}$.

It is obvious that in order to achieve an optimum benefit from this technology, a large amount of detailed design must be performed. The airfoils used on the wing and nacelle are certain to have a significant effect on the efficiency gains accomplished via the engine mounting scheme explored in this paper. Structural optimization and detailed mission requirements are also sure to play their role, and must both be explored in higher detail. Since detailed optimization was not the goal, the study was performed with general components, and the results achieved are not necessarily indicative of the highest possible level of performance benefits of this type of a nacelle mounting.

From the flow visualizations obtained in the CFD portion of the study, it can be seen that the superposition of the nacelle body and flow-field generated ahead of it has a significant impact on the performance of the wing. By beneficially interacting with an existent zone of supersonic flow, the nacelle allows for a weaker shock, and therefore a smaller pressure increase to occur after the suction zone on the wing's upper surface. This increase in area of high-speed flow and decrease in strength of supersonic shock are the basis the drag-reducing and liftimproving behavior of conventional supercritical airfoils. Finally, it also can be seen that although the nacelle body produces some beneficial results when interacting with an already-formed area of supersonic flow, care should be exercised in nacelle positioning to avoid creating a secondary area of high-speed flow via acceleration in the interface between the nacelle and the wing's upper surface.

While more detailed research is likely to be necessary to fully appreciate the effects of this nacelle-wing interaction, it has been shown that the basic technology seems to have some promise. An optimized nacelle location 
was achieved and a set of real-life performance benefits were demonstrated. Additionally, a set of important, yet difficult to quantify benefits, such as increased internal space afforded to the passengers, and the structural synergy of the landing gear, wing and nacelle mount are associated with this method of engine mounting are all sure to benefit a prospective business jet design. Finally, a set of tools have been created and a large amount of work has been conducted to enable further and more in-depth research into the subject.

\section{References}

1 Fujino, M., Kawamura, Y. Honda Giken Kogyo Kabushiki Kaisha. Method for reducing wave resistance in Airplane. United States Patent \#6,308,913. October 30, 2001

2 Bertin, J. J. and Smith, M. L. Aerodynamics for Engineers, $2^{\text {nd }}$ ed. Prentice-Hall, Englewood Cliffs, New Jersey. 1989., 456

3 TRANAIR Users's Manual. 91

4 Drela, M. and Giles, M. B. ISES: a two-dimensional viscous aerodynamic design and analysis code. American Institute of Aeronautics and Astronautics (AIAA). (Paper No. 87-0424). 1987.

5 MISES brochure, Analytical Methods, Inc., Redmond, WA.

6 Terry, Eric L.N. Extension of the aerodynamic program MSES for the simulation of boundary layer suction. Delft University of Technology. November 2004

7 Ibid, 13

8 Meyers, M. K. Aircraft Data Base System: Presentation of Select Weight, Design and Dimensional Data in Graphical and Table Formats - Documentation and User's Guide. Penthouse Productions. April 1988

9 XIXtrFun ${ }^{\mathrm{TM}}$ Extra Functions for Microsoft Excel. Advanced Systems Design and Development. <www.xlxtrfun.com>. 28 Jan. 2006

10 Roache, P.J., Ghia, K., and White, F. Editorial Policy Statement on the Control of Numerical Accuracy. ASME Journal of Fluids Engineering, Vol. 108, No. 1., March 1986, p. 2.

11 Gisin, Yevgeniy. M., Wing-Nacelle Assembly Multidisciplinary Performance Optimization, California Polytechnic State University, San Luis Obispo. March 2006 